\title{
Aspetti dottrinali delle nuove omelie di Origene sui Salmi: le tematiche cristologiche a confronto col Perì Archôn ${ }^{*}$
}

\author{
Lorenzo Perrone \\ "ALMA MATER STUDIORUM" - UNIVERSITÀ DI BOLOGNA \\ lorenzo.perrone@unibo.it
}

Resumen: Para Orígenes, tal como lo muestran muchos de sus escritos y sus numerosas citaciones, el libro de los Salmos es importante no solo desde el punto de vista moral y espiritual, sino también desde la perspectiva doctrinal. Las homilías sobre los Salmos recientemente descubiertas en el códice de Munich (Codex Monacensis Graecus 314) lo confirman de modo claro. No por casualidad un fragmento de la segunda homilía sobre el Sal. 15 fue integrado por Pánfilo en su Apología de Orígenes para probar la conformidad del maestro de Alejandría con la fe de la Iglesia respecto de la resurrección corporal. Como regle general, los nuevos sermones contribuyen a profundizar los aspectos espirituales como doctrinales del pensamiento de Orígenes, de acuerdo al modelo que ve la teología como la consecución de la práctica. Los esfuerzos intelectuales para alcanzar la verdad de Dios y del hombre deben siempre ser preparados por la ejercitación de la virtud y por el progreso espiritual. Sobre esta base, junto con los conocidos tópicos de la teología de Orígenes, en los nuevos sermones encontramos muchas ideas originales. Su amplio espectro se puede comparar con los temas del Perì Archôn. En la séptima Homilía sobre el Salmo 77 encontramos un pasaje que quizá ofrece lo que parece ser la mejor explicación para el título del tratado dogmático de Orígenes: según él, hay "principios", es decir, doctrinas referidas al Padre, al Hijo, al Espíritu Santo, a la Iglesia y a los ángeles, que, como fuentes, fluyen hacia el único "río" de Cristo y de su Iglesia. Otro punto que asoma como un tema frecuente consiste en la visión de la "divinización". En la homilía sobre el Salmo 81, Orígenes, ampliando aún más sus puntos de vista anteriores, insiste explícitamente en la idea de la deificación como un proceso que involucra a todo el hombre: no solo su espíritu y el alma, sino también su cuerpo. Para limitar este análisis a la cristología, las dos homilías sobre Sal 15 desarrollan la doctrina de la «descenso» (katabasis) del Logos encarnado en el mundo y el inframundo (Hades) y, en un movimiento contrario, su "ascenso" (anabasis) al cielo. Orígenes describe el proceso de encarnación, insistiendo, por una parte, en la comunión plena entre el Hijo 
y el Padre y, por otra, haciendo hincapié en la plena humanidad del Hijo. El cuerpo resucitado de Cristo, que asciende al Padre a través de los cielos es un "cuerpo terren", que todavía presenta las huellas de su pasión.

Palabras clave: Orígenes, cristología, Salmos, Codex Monacensis Graecus 314.

Abstract: For Origen the book of the Psalms, as shown by his many writings and the numerous quotations, is important not only from a moral and spiritual point of view but also in a doctrinal perspective. The newly discovered Homilies on the Psalms in the Munich codex (Codex Monacensis Graecus 314) provide a significant confirmation to it. Not incidentally a fragment of the 2nd Homily on Ps. 15 was inserted by Pamphilus in his Apology of Origen in order to prove the conformity of the Alexandrian master with the beliefs of the Church concerning the resurrection of the body. As a general rule, the new sermons contribute to deepen both the spiritual and doctrinal aspects of Origen's thought, complying with a pattern that views 'theology' as the accomplishment of 'practice'. Intellectual efforts towards reaching the truth on God and man must always be prepared by virtuous training and by spiritual progress. Against this background, together with well-known topics of Origen's theology, in the new sermons we find lot of original insights. Their wide spectrum can be compared with the topics of the Perì archôn. In the 7th Homily on Ps. 77 we find a passage perhaps delivering what seems to be the best explanation for the title of Origen's dogmatic treatise: according to it, there are 'principles', that is doctrines, with regard to the Father, the Son, the Holy Ghost, the Church and the angels, that like sources flow into the only 'river' of Christ and his Church. Another point which surfaces as a frequent theme consists in the view of 'deification'. In the Homily on Ps. 81 Origen, by further enlarging his previous views, explicitly insists on the idea of deification as a process involving the whole man: not only his spirit and soul, but also his body. To limit the present analysis to Christology, the two Homilies on Ps. 15 develop the doctrine of the 'descent' (katabasis) of the Logos incarnate into the world and the underworld (Hades) and in an opposite move his 'ascent' (anabasis) to heaven. Origen describes the process of incarnation by insisting, on the one hand, on the full communion between the Son and the Father and, on the other hand, by stressing the full humanity of the Son. The resurrected body of Christ who ascends to the Father through the heavens is an 'earthly body' still presenting the traces of his passion.

Keywords: Origen, christology, Psalms, Codex Monacensis Graecus 314. 
¡Biblia, mi noble Biblia, panorama estupendo, en donde se quedaron mis ojos largamente, tienes sobre los Salmos las lavas más ardientes y en su río de fuego mi corazón enciendo!

Gabriela Mistral

\section{Premessa: Origene 'archetipo' dell'esegesi patristica dei Salmi}

Nel cristianesimo antico l'esegesi dei Salmi ha rappresentato un luogo privilegiato per l'approfondimento dottrinale, accanto alla loro interpretazione in chiave prevalentemente morale e spirituale ${ }^{1}$. In particolare, $\mathrm{i}$ Salmi sono stati sfruttati per il discorso cristologico nonché per quello ecclesiologico, come evidenzia la magistrale 'sintesi' elaborata da Agostino nelle Enarrationes in Psalmos grazie al riconoscimento del locutore dei Salmi nel Cristo in quanto capute corpus, cioè rispettivamente la persona del Figlio, Dio e uomo, e la chiesa suo corpo mistico ${ }^{2}$. Origene si colloca agli inizi di questi sviluppi esegetici, dal momento che non solo si riallaccia alle indicazioni già contenute nel Nuovo Testamento riguardo ai due principali registri interpretativi del Salterio ${ }^{3}$, ma conferisce loro un'applicazione organica che va ben al di là dell'uso in chiave profetico-tipologica di un Giustino, dell'esegesi cristologica di un Ippolito o dell'approccio allegorico su scala ancora ridotta di un Clemente Alessandrinot. L'importanza

Prolusione al IX Seminario de Estudios Patrísticos, Facultad de Teología y Facultad de Filosofía, Pontificia Universidad Católica de Chile (Santiago de Chile, 24-27 settembre 2013). Desidero ringraziare Samuel Fernández per il generoso invito e l'ospitalità fraterna, estendendo la mia profonda riconoscenza ai colleghi cileni e argentini per l'indimenticabile esperienza di amicizia e scambio intellettuale.

1 Per una visione d'insieme, cf. M. J. Rondeau, Les commentaires patristiques du Psautier (III - V' siècles). I: Les travaux des Pères grecs et latins sur le Psautier. Recherches et bilan; II: Exégèse prosopologique et théologie (Roma 1982, 1985). Si veda anche l'introduzione di Manlio Simonetti a Sant'Agostino. Commento ai Salmi, a cura di M. Simonetti (Milano 1989).

2 M. Fiedrowicz, Psalmus Vox Totius Christi. Studien zu Augustins Enarrationes in Psalmos (Freiburg i. Br. 1997).

3 Sull'impiego neotestamentario si veda, ad es., M. Simonetti, I "salmi” nel Nuovo Testamento, in Orpheus, 9 (1988) 1-20.

4 L'interesse di Clemente per i Salmi è attestato, in particolare, dagli Stromati e dalle Ecloghe profetiche, su cui si veda da ultimo M. CAMBE, Avenir solaire et angélique des justes: Le psaume 19 (18) commenté par Clément d'Alexandrie (Strasbourg 2009). 
fondativa della sua esegesi è attestata, del resto, non solo dalla complessa documentazione dell' Esapla, arricchita -al dire di Eusebio- di ben tre altre colonne per le 'edizioni' greche del testo dei Salmi', ma soprattutto dalla straordinaria ampiezza del suo progetto letterario. Il commento al Salterio accompagna infatti le diverse fasi della vita di Origene, a cominciare dal periodo alessandrino fino ad arrivare praticamente alla conclusione del suo impegno esegetico nel soggiorno a Cesarea.

Come è noto, non è facile tracciare un quadro preciso in proposito, per l'incertezza dei dati a nostra disposizione ${ }^{6}$. La Lettera 33 di Gerolamo, contenente il catalogo degli scritti di Origene tratto dalla Vita di Panfilo di Eusebio di Cesarea, ci fa intravedere comunque lo sforzo ripetuto dell'Alessandrino per offrire commentari adeguati, più o meno estesi, del libro più noto e familiare dell'Antico Testamento. Se ci atteniamo alla ricostruzione proposta da Pierre Nautin, a un primo Commento ai Salmi 1-25, redatto ancora nel periodo alessandrino ${ }^{7}$, ha fatto seguito un più corposo commento scritto a Cesarea e formato da almeno una quarantina di tomoi, secondo l'elenco di Gerolamo, peraltro presumibilmente incompleto ${ }^{8}$. In aggiunta, l'Alessandrino compose an-

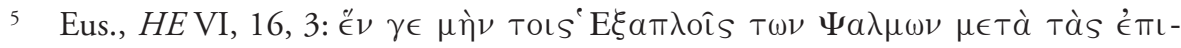

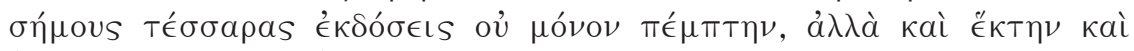

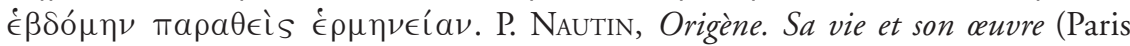
1977) 312-314, ritiene che Eusebio abbia frainteso le indicazioni di Origene aggiungendo una 'settima' versione. Accettano invece la testimonianza eusebiana A. Grafton-M. Williams, Christianity and the Transformation of the Book (Cambidge, Ma.-London 2006) 89-90, 206-207.

6 L'ipotesi ricostruttiva più organica, ma non da tutti condivisa, è quella avanzata da Nautin, Origène, cit., 261-292. Un riesame critico delle posizioni di Nautin è stato fatto, tra gli altri, da M. J. Rondeau, Les commentaires patristiques du Psautier (III $I^{e} V^{E}$ siècles), cit., I, 45 ss. Per una prima sintesi, cf. E. PrinzIVAlli, Salmi (scritti esegetici sui), in A. Monaci Castagno (a cura di), Origene. Dizionario: la cultura, il pensiero, le opere (Roma 2000) 422-424.

7 Si trattò, a quel che dichiara lo stesso autore, della sua prima opera (cf. CPs 1-25 Prol in Epifanio, Panarion 64.5-7), su cui si veda É. JunOD, Du danger d'écrire, selon Origène, in Origeniana Decima. Origen as Writer. Papers of the 10th International Origen Congress, ed. by S. KaczmareK - H. Pietras (Leuven 2011) 91-108. Le riserve sulla priorità del commentario alessandrino espresse da R. Heine, Origen: Scholarship in the Service of the Church (Oxford 2010) 117, non appaiono persuasive proprio alla luce della dichiarazione di Origene.

8 Ad es., nell'Ep. 33 manca l'indicazione di un tomus su Sal 47, attestato invece da CC VII, 31 (182, 3-9), dove Origene ricorda di avervi trattato il tema della

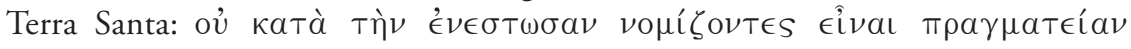


che degli Scolî sui Salmi, di non facile collocazione cronologica rispetto al commentario maggiore, anche se per Nautin andrebbero situati sul finire della vita e sarebbero da considerare quale soluzione alternativa al compito impossibile di commentare l'intero libro 9 . Al contrario, per Franz Xaver Risch si sarebbe trattato di lavori preparatori per futuri commentari ${ }^{10}$. Infine, anche il terzo genere esegetico praticato dall'Alessandrino ha dato luogo a un insieme imponente di 120 Omelie sui Salmi, sempre attenendoci alla lista di Gerolamo ${ }^{11}$.

Al di là di questa immensa impresa esegetica -senza dubbio la più significativa di tutta l'attività letteraria dell'Alessandrino per numero ed entità degli scritti- non bisogna dimenticare l'uso che Origene ha fatto del Salterio nell'insieme della sua opera. Chi ha familiarità con il repertorio di Biblia Patristica sa che le citazioni dei Salmi superano di gran lunga quelle di ogni altro libro sia dell'Antico sia del Nuovo Testamento, se si esclude il Vangelo di Matteo ${ }^{12}$. Ciò spiega verosimilmente l'assenza di indagini in proposito, per la difficoltà ad approfondire i percorsi esegetici dell'Alessandrino alla luce di un'attestazione tanto ricorrente e capillare. Un'eccezione al riguardo è rappresentata proprio dal Perì Archôn, che è stato oggetto di uno studio recente sull'utilizzo dei Salmi

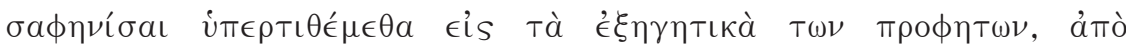

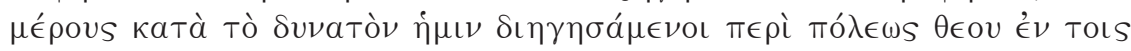

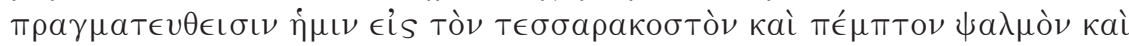

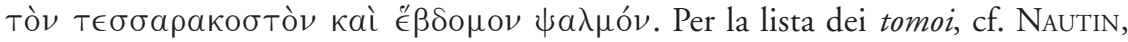
Origène, cit., 249.

9 Cf. Ibid., 292: "Vers la fin de sa vie, à la demande peut-être d'un ami, il se remit à la tâche. Comme il ne pouvait plus songer à compléter le commentaire de Césarée à cause du travail écrasant que cela aurait exigé, il composa un nouvel ouvrage, très court, sous la forme de Scolies sur le Psautier, dans lequel il se bornait à donner quelques indications sur les versets ou les mots qui appelaient une remarque. De cette manière, il put enfin achever le psautier".

10 Per un nuovo esame del problema degli Scolî si veda F.X. Risch, Die Scholien des Origenes zu den Psalmen. Bemerkungen zur zweiten Randkatene im Codex Vindobonensis theol. gr. 8 (di prossima pubblicazione in Adamantius 20 [2014]). Cf. anche ID., Zur lateinischen Rezeption der Scholia in Psalmos des Origenes (di prossima pubblicazione in Origeniana Undecima).

11 Per la lista delle omelie, cf. Nautin, Origène, cit., 258.

12 Cf. Biblia Patristica. Index des citations et allusions bibliques dans la littérature patristique (Paris 1980, 1991). I Salmi vi occupano 45 pp. rispetto alle $57 \mathrm{di} \mathrm{Mt}$. 
ad opera di John McGuckin ${ }^{13}$. I suoi risultati confermano l'impressione che il ricorso al Salterio assuma un valore fondamentale per il pensiero di Origene, offrendogli alcuni dei nuclei scritturali più consistenti e costanti per tutta una serie di trattazioni esegetiche e dottrinali ${ }^{14}$. A mia volta, ho potuto verificare il peso normativo del riferimento ai Salmi per la teologia della preghiera sviluppata dall'Alessandrino nel Perì Euchês e nel resto della sua opera ${ }^{15}$.

L'apporto esegetico-dottrinale delle omelie del Cod. Graec. 314

Le perdite subite dal vastissimo corpus degli scritti di Origene sui Salmi sono tra le più gravi ed estese. Purtroppo, poco ci è rimasto dei grandi tomo $i$, pervenuti perlopiù tramite le catene in frammenti di ridotta estensione (e in molti casi accompagnati dall'incertezza sull'effettiva attribuzione all'Alessandrino), benché gli ampî excerpta dai commenti su Sal 4 e 118 consentano di ridisegnare parzialmente la loro fisionomia robusta e complessa ${ }^{16}$. Nello stato tuttora incerto della tradizione catenaria, in assenza di edizioni più attendibili di quelle di cui disponiamo attualmente, un punto fermo era rappresentato finora dalla traduzione latina delle nove Omelie sui Salmi 36, 37, 38 ad opera di Rufino, con-

13 J.A. McGuckin, Origen's Use of the Psalms in the Treatise On First Principles, in Meditations of the Heart: The Psalms in Early Christian Thought and Practice. Essays in Honour of Andrew Louth, ed. by A. Andreopoulos, A. Casiday, C. Harrison (Turnhout 2011) 97-118.

14 McGuckin, Origen's Use of the Psalms in the Treatise On First Principles, cit., 117: "The Psalms hold immense significance for him. They are of sufficient weight to 'stand for' the whole Old Testament revelation if necessary. Origen feels that they possess a dominating force of tradition both for the simpliciores, and for the more educated members of the Christian community (including his own schola). He uses the Psalms often to balance his reliance on those other 'first fruits' of the scriptural revelation, Paul and John. He sees the Psalms as distinctive in the Old Testament as being able to penetrate the shadows much more effectively, and frequently to serve as the locus of the direct teachings of the Logos himself. Accordingly, the 'Prophet of the Psalms' can sometimes see acutely, penetratingly, into the greatest of all mysteries of the cosmos".

15 L. Perrone, La preghiera secondo Origene. L'impossibilità donata (Brescia 2011) spec. le pp. 438-441, 449-451, 454-458.

16 Si veda rispettivamente Origène. Philocalie 21-27: Sur le libre arbitre, introd., texte, trad. et notes par É. Junod (SC 226), Paris 1976, 94-102, 234-267 (= Phil 26, 1-8 su Sal. 4, 6); e La chaîne palestinienne sur le Psaume 118 (Origène, Eusèbe, Didyme, Apollinaire, Athanase, Théodoret), a cura di M. Harl, I-II (SC 189, Paris 1972). 
fortata da un esiguo numero di frammentari catenari greci ${ }^{17}$. Tuttavia, dall'aprile dello scorso anno la nostra documentazione si è arricchita in maniera sostanziosa grazie all'eccezionale scoperta di ventinove omelie di Origene nel Cod. Graec. 314 della Bayerische Staatsbibliothek di Monaco da parte di Marina Molin Pradel, contenente fra l'altro il testo originale delle Omelie I-IV sul Salmo $36^{18}$.

Come per le Omelie sul Salmo 36, la lista dei sermoni nel manoscritto monacense, datato all'inizio del XII secolo, corrisponde largamente all'elenco della Lettera 33 di Gerolamo. Infatti, le apparenti discrepanze si lasciano quasi tutte sciogliere alla luce delle presumibili vicissitudini della tradizione manoscritta. La collezione comprende così la seguente serie di sermoni: due omelie su Sal 15, quattro su Sal 36, due su Sal 67, tre su Sal 73, una su Sal 74, una su Sal 75, quattro su Sal 76, nove su Sal 77, due su Sal 80, una su Sal 81 ${ }^{19}$. La II Omelia sul Salmo 15 è altresì attestata da un ampio frammento latino incluso da Panfilo nell'Apologia di Origene ${ }^{20}$, mentre echi più o meno trasparenti si notano in alcuni dei Tractatus in Psalmos di Gerolamo, a suo tempo rivendicati in blocco da Vittorio Peri come opera di Origene ${ }^{21}$.

Dagli estratti del tomus su Sal 4, tramandati nella Filocalia e incentrati sulla questione dei veri 'beni', possiamo intuire quanto impegnata sul piano filosofico e teologico potesse essere all'occorrenza l'interpretazione

17 Origene. Omelie sui Salmi, Homiliae in Psalmos XXXVI - XXXVII - XXXVIII, a cura di E. Prinzivalli, Firenze 1991; Origène. Homélies sur les Psaumes 36 à 38, texte critique établi par E. Prinzivalli, Intr., trad. et notes par H. Crouzel et L. BréSARD (SC 411, Paris 1995).

18 Per una descrizione delle modalità della scoperta e il processo di attribuzione si veda M. Molin Pradel, Novità origeniane dalla Staatsbibliothek di Monaco di Baviera: il Cod. graec. 314, in Adamantius, 18 (2012) 16-40.

19 Rimando ai miei seguenti contributi per una descrizione più dettagliata: Riscoprire Origene oggi: prime impressioni sulla raccolta di omelie sui salmi nel Codex Monacensis Graecus 314, in Adamantius, 18 (2012) 41-58; Rediscovering Origen Today: First Impressions of the Newly Discovered Collection of Homilies on the Psalms, in Studia Patristica LVI/4, ed. by M. VInZENT (Leuven 2013) 103-122; Origenes rediuiuus: La découverte des homélies sur les Psaumes dans le Cod. Gr. 314 de Munich, in Revue des Études Augustiniennes et Patristiques, 59 (2013) 55-93.

20 Panfilo, Apol. 142-145 in Pamphile et Eusèbe de Césarée. Apologie pour Origène, Texte critique, traduction et notes par R. Amacker et É. JunOd (SC 464, Paris 2002) 228-232.

21 V. Peri, Omelie origeniane sui Salmi. Contributo allidentificazione del testo latino (Città del Vaticano 1980). 
origeniana del Salterio, anche se ciò non doveva valere in tutti i casi. Vi erano infatti salmi che attiravano in primis, se non programmaticamente, una spiegazione morale, come vediamo in particolare, per la nostra serie di sermoni, dal prologo dell' Omelia I sul Salmo 36. L'Alessandrino precisa qui la sua ratio ermeneutica, prefigurando tre possibili approcci interpretativi che, a seconda dei luoghi commentati, comportano a) un'esegesi profetico-tipologica dei Salmi; b) una spiegazione mistico-allegorica; c) un'applicazione morale. Nel caso di Sal 36 si tratta di un

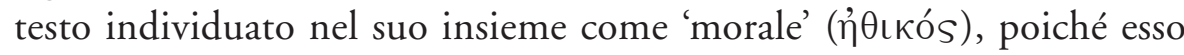
possiede una valenza terapeutica che si esplica da un lato nel rimprovero per i peccati commessi e dall'altro nella sollecitazione a convertirsi e praticare una condotta retta ${ }^{22}$. Rufino, nel presentare la propria antologia omiletica, estende la caratterizzazione in senso morale ai Sal 37 e 38, rispecchiando fedelmente il tenore del ciclo di omelie che traccia l'itinerario spirituale dalla conversione alla perfezione ${ }^{23}$. Sotto questo punto di vista la serie dei nove sermoni su Sal 36-38 si ricollega ad altri salmi definiti ugualmente da Origene come 'morali' - quali Sal 111, 112

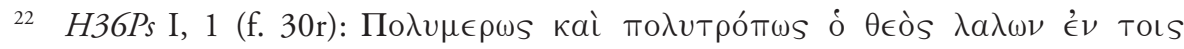

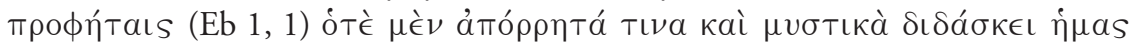

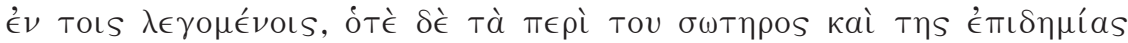

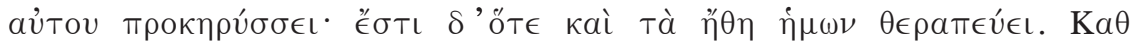

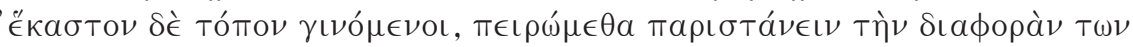

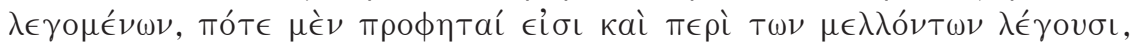

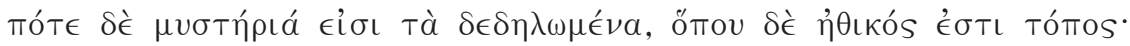

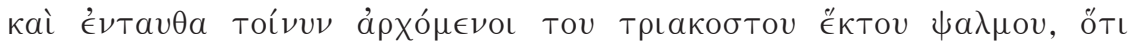

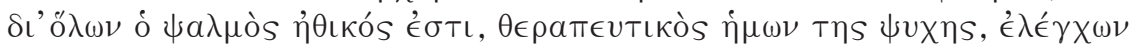

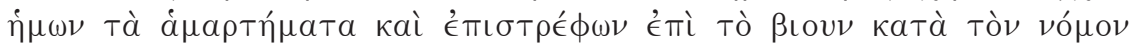
(trascrizione a cura di Emanuela Prinzivalli). Si noti che la prima delle due formulazioni sui tre sensi scritturistici inverte l'ordine fra il primo e il secondo proposto nella seconda. Circa l'approccio esegetico di H36-38Ps si veda K.J. Torjesen, Hermeneutical Procedure and Theological Method in Origen's Exegesis (Berlin-New York 1986); E. Prinzivalli, Vinea spiritualis intellegentiae. L'interpretazione omiletica dei salmi in Origene. Un'indagine a partire dalle omelie sui salmi 3637 38, in Annali di storia dell'esegesi, 7 (1990) 397-416.

23 Nella prefazione alla traduzione, Rufino sembra riscrivere la dichiarazione ermeneutica di Origene: Quoniam tricesimi sexti et tricesimi septimi et tricesimi octaui psalmi expositio tota moralis est, instituta quaedam uitae emendatioris ostendens, et nunc conuersionis ac paenitentiae, nunc purgationis et profectuum semitam docet (Origene. Omelie sui Salmi, ed. Prinzivalli, 26, 1-5). 
e 118, che essendo tutti alfabetici intrecciano più strettamente l'istanza etica con quella didascalico-pedagogica ${ }^{24}$.

Essi cristallizzano quindi il primo stadio del progresso spirituale che per l'Alessandrino -come vediamo nell' Omelia I sul Salmo 77- conduce dall'osservanza della legge di Cristo alla comprensione del suo 'insegnamento'. In questo sermone egli insiste sull'"ordine' ( $\tau a ́ k \iota)$ che bisogna rispettare per pervenire alla 'teologia' $(\theta \in o \lambda o \gamma(\hat{a} \alpha)$, secondo una bipartizione fra etica e teoria ispirata dalla sequenza evangelica fra discorso sulla montagna e insegnamento in parabole, ma che richiama comunque, per il suo esito ultimo, il paradigma più noto di morale-fisica-epoptica descritto nel prologo al Commento al Cantico dei Cantici ${ }^{25}$. Nel primo dei nove sermoni su Sal 77 Origene fa valere il richiamo all'ortoprassi' come la via e il metodo corretti per giungere all'ortodossia' in polemica con le dottrine degli eretici: la loro ricerca della verità, per quanto intensa e faticosa possa essere, segue in realtà un percorso sbagliato, perché non si attiene alla premessa posta necessariamente dalla vita morale e dall'adesione di fede prima di affrontare il compito dell'investigazione teologica ${ }^{26}$.

Pur tenendo conto delle precisazioni di Origene e di Rufino, sarebbe riduttivo operare una distinzione troppo netta fra interpretazione morale e spiegazione dottrinale, essendo i due aspetti -come abbiamo visto- strettamente interdipendenti fra loro. In ogni caso le estrapolazioni teologiche ricavate dall'Alessandrino nell'interpretare i nostri salmi sono numerose

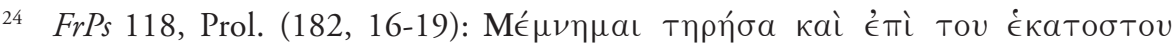

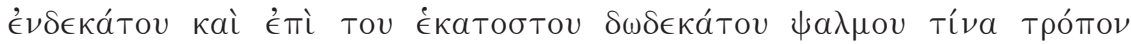

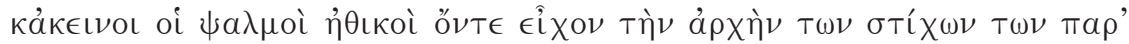

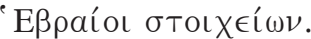

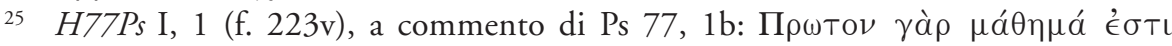

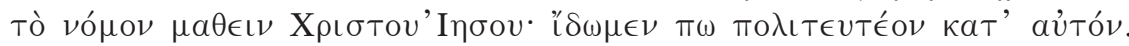

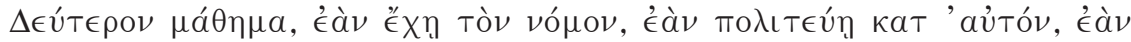

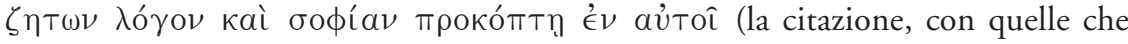
seguono, è tratta dalla mia edizione provvisoria). Altrove, come in CIo I, 30, 208, la bipartizione fra 'etica' e 'teologia' verrà riproposta a partire dall'equazione simbolica rispettivamente col 'pane' e il 'vino'. Anche H80Ps I, 4 (f. 336r) prefigura

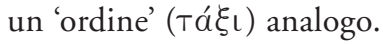

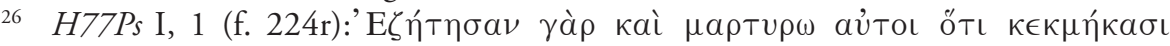

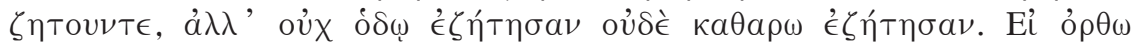

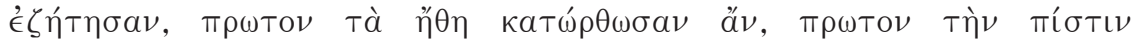

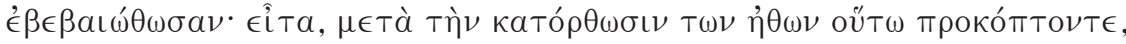

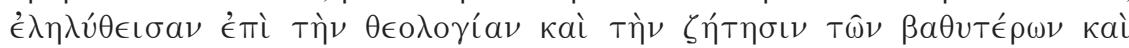
$\mu v \sigma \tau \iota \kappa \omega T \epsilon ́ \rho \omega \nu$. 
e assai significative. Si può dire che la sua esegesi non solo comporta implicazioni cristologiche ed ecclesiologiche -conformemente al duplice approccio richiamato in apertura-, ma investe più ampiamente un esteso spettro tematico, a cominciare dalla riflessione sull'esercizio teologico fino alle concezioni su Dio, il Figlio, lo Spirito, e al pensiero su cosmo, angeli e demoni, sull'uomo e il suo destino. In questo senso le nuove omelie rispecchiano molte delle tematiche dottrinali affrontate nel Perì archôn. E forse non è un caso se vi si ritrova una riflessione sulle à $\rho \chi a i ́$, che sembrerebbe offrire la migliore spiegazione del titolo scelto da Origene per la sua opera dogmatica e risentito come ambiguo già da Rufino ${ }^{27}$. Essa compare nell' Omelia VII sul Salmo 77 e il contesto in cui viene formulata mostra nuovamente la continuità di pensiero col discorso prima accennato su etica e teologia. L'Alessandrino sviluppa la spiegazione delle piaghe d'Egitto, che è il tema dell'omelia, commentando Sal 77, 44: "Cambiò in sangue

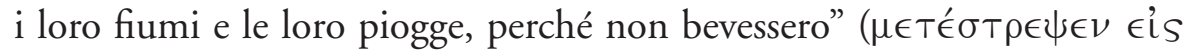

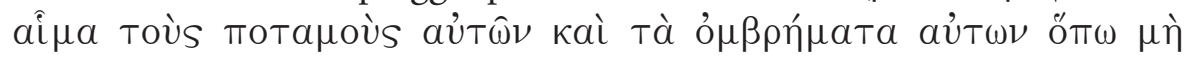
$\pi i ́(\omega \sigma \nu)$. Per antitesi con l'acqua degli Egiziani, egli introduce una riflessione sui 'fiumi' dei santi, già abbozzata in parte nella Omelia III sul Salmo 76 e ricca di risonanze in molti dei suoi scritti ${ }^{28}$. Il discepolo di Gesù è colui dal quale scorre una fonte di acqua viva $(\mathrm{Gv} 4,14)$ : non un 'fiume' unico, bensì più 'flumi', cioè i 'teoremi' e le 'dottrine' che scaturiscono da ciascuno di essi. Origene lo esemplifica negli apostoli Paolo e Pietro, dai quali scorrono fiumi che, come quelli del paradiso terrestre, scorrono verso i quattro punti cardinali ${ }^{29}$.

27 Come si evince dalla traduzione alternativa in Ruf., Praef. Prin 3: istos, quos nunc exigis ut interpreter, id est ПEPI APX $\Omega \mathrm{N}$, quod uel De principiis uel De principatibus dici potest.

28 Cf. H76Ps III, 4 (ff. 201r-v); CIo VI, 42, 219.

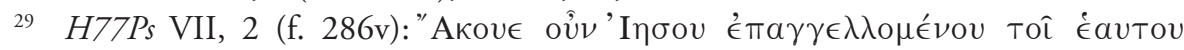

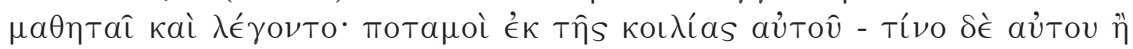

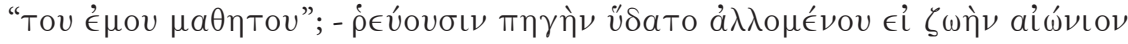

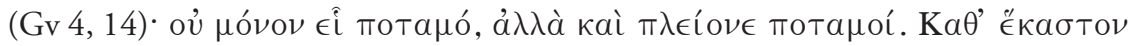

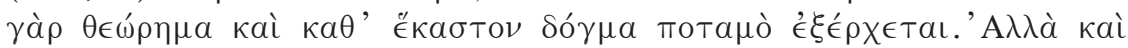

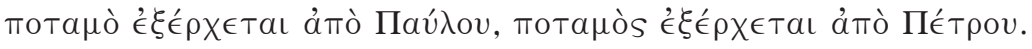




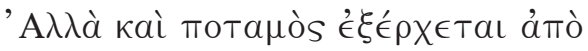

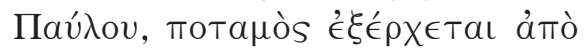

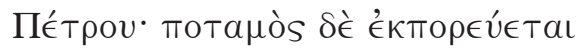

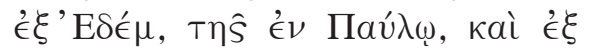

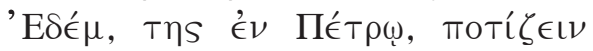

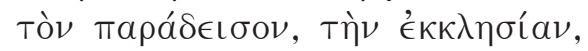

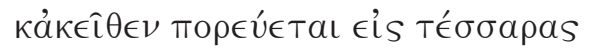
àpxás (Gn 2, 10).

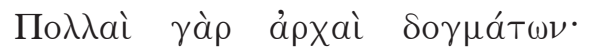

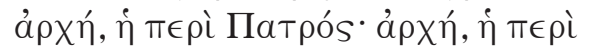

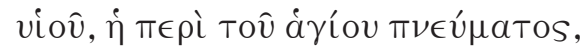

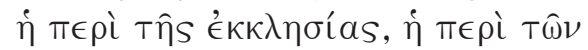

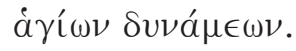

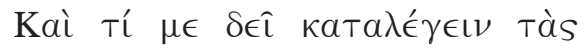

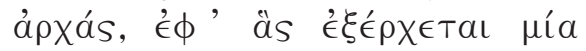

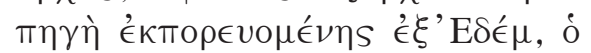

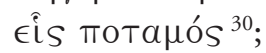

Ma vi è un fiume che fuoriesce da Paolo ed uno che fuoriesce da Pietro: Un fume scorre dall'Eden, l'Eden di Paolo, e uno dall'Eden di Pietro, per irrigare il paradiso, la Chiesa, e da lì si diffonde verso i quattro principi (Gn $2,10)$.

Molti sono infatti i princìpi delle dottrine: un principio è quello relativo al Padre; un principio è relativo al Figlio, uno allo Spirito santo, uno alla Chiesa, uno alle potenze sante.

Ma quale bisogno c'è di elencare i principi, verso i quali scorre l'unica sorgente che scaturisce dall'Eden, l'unico fiume?

Come chiarisce il prosieguo dell'interpretazione, questo "unico fiume" non è altri che il Cristo. Origene lo chiarisce servendosi di Sal 45,

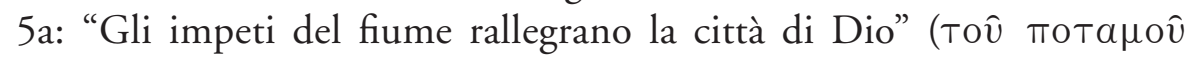

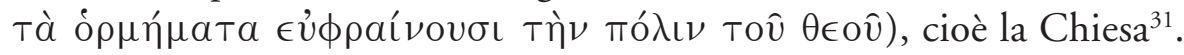
L'abbondanza dell'acqua che da Cristo si riversa nei molteplici fiumi dei suoi fedeli ha la propria sorgente nella presenza effusiva del Padre in Lui, secondo quanto l'Alessandrino dichiara commentando nuovamente altrove Sal $45,5 \mathrm{a}^{32}$.

$30 \quad$ Ibid. (ff. 286v-287r).

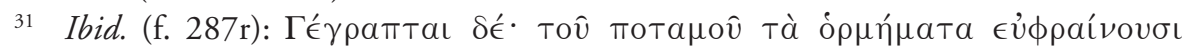

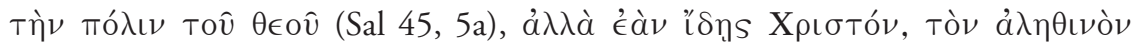

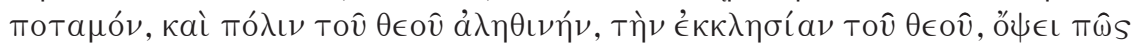

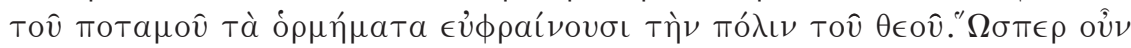

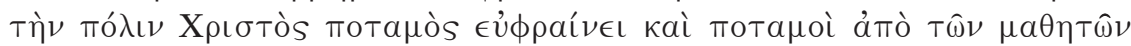

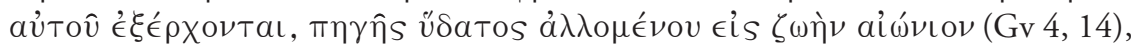

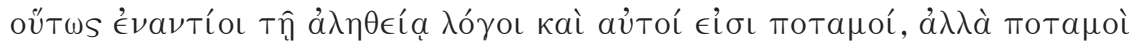

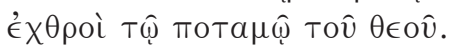

32 Egli ha commentato ripetutamente Sal 45, 5a. Fra i passi più significativi si veda

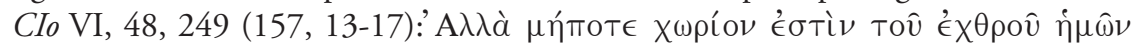

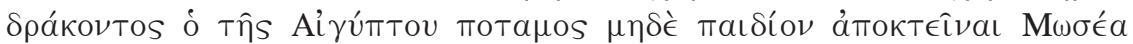

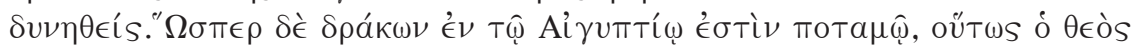

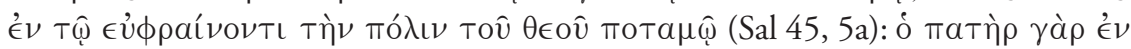




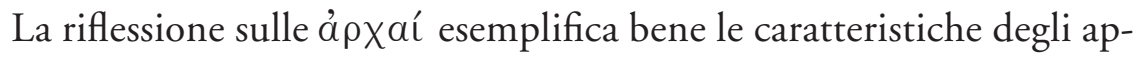
porti dottrinali che ci vengono dalle nuove omelie: mentre per molti aspetti esse si collocano in una linea di continuità con il pensiero di Origene-come ci appare dal Perì archôn e dall'insieme degli scritti-, per altro verso non mancano di manifestare spunti innovativi e accenti diversificati. A titolo d'esempio, avendo io cercato di ripercorrere il discorso origeniano sulla preghiera nel suo complesso, non ho potuto fare a meno di notare, con sorpresa ed interesse, delle argomentazioni inedite che contribuiscono ad arricchire una riflessione già altrimenti intensa $\mathrm{e}$ articolata. Esse attengono in particolare, come si vedrà a breve, le enunciazioni cristologiche, poiché l' Omelia I sul Salmo 15 estende il rapporto di dipendenza orante tra il Figlio e il Padre, oltre che naturalmente alla persona divino-umana dell'Incarnato, alla stessa Sapienza $(\Sigma o \phi i ́ a)^{33}$. Inoltre, nell' Omelia I sul Salmo 76, Origene torna a riproporre la morte in croce di Gesù quale espressione di preghiera, in conformità con l'esegesi offerta nelle Series in Matthaeum ${ }^{34}$, sottolineando però ulteriormente la portata simbolica universale della braccia aperte del Crocifisso, segno della 'coestensività' della sua anima su tutto il mondo ${ }^{35}$. Ancor più rilevante è forse la retractatio proposta dall'Alessandrino nell' Omelia VIII sul Salmo 77 riguardo alla dottrina degli angeli, che egli riconnette abitualmente all'interpretazione di Dt 32, 8-9. In questa occasione egli tiene a confessare all'uditorio di aver cambiato idea in proposito, essendosi ormai convinto che l'assegnazione degli angeli alle diverse nazioni abbia avuto luogo non più dopo la dispersione delle genti per la torre di

T⿳̂ vị̣̂; CCt III (221, 8-10): Quid enim imbribus opus est, ubi flumen Dei laetificat ciuitatem (Sal 45, 5a), ubi in corde uniuscuiusque credentium fons aquae uiuae fit salientis in uitam aeternam (Gv 4, 14)?; HEz XIII, 4 (449, 27-31): Et scio aliud flumen, cuius impetus laetificat ciuitatem Dei, iuxta Psalmistae nocem dicentis: fluminis impetus laetificat ciuitatem Dei (Sal 45, 5a). Vis audire, quis est iste fluvious, cuius impetus laetificat ciuitatem Dei? Iesus Christus Dominus noster est fluuius.

33 H15Ps I, 3 (ff. 4r-v). Cf. infra n. 56.

34 Cf. CMtS 138-140 e il mio articolo: La morte in croce di Gesù epifania divina del mistero del Logos fatto carne (Origene, Commentariorum Series in Matthaeum 138140), in Adamantius 16 (2010) 286-307.

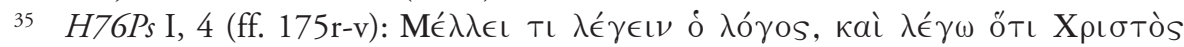

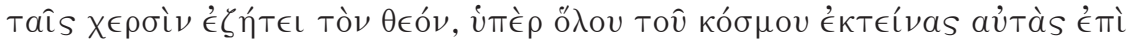

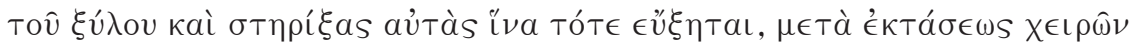

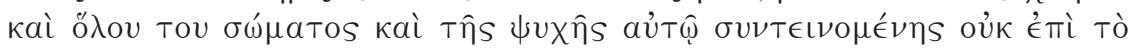

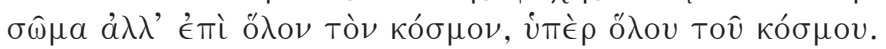


Babele bensì a seguito dell'elezione d'Israele come popolo di Dio con il suo esodo dall'Egitto ${ }^{36}$.

Per completare questi cenni sommari sui risvolti dottrinali, si dovrà prendere atto dell'importanza singolare che riveste il tema della deificazione. Sebbene esso si presti ad un approfondimento, per così dire, dovuto, a causa dell'esegesi di Sal 81, proprio questo testo-chiave, insieme ad altri passi scritturistici, sono l'indice di una presenza molto più pervasiva. Di fatto il motivo della deificazione ha una ricaduta talmente frequente nei nostri sermoni da doverlo considerare un elemento che innerva in generale l'interpretazione origeniana del Salterio ${ }^{37}$. Fra l'altro, esso porta nell' Omelia sul Salmo 81 al riconoscimento forse più esplicito dell'Alessandrino sul fatto che anche il corpo sia coinvolto nel processo di divinizzazione dell'uomo, insieme all'anima e allo spirito ${ }^{38}$. Anche

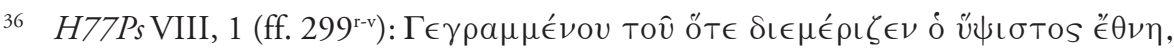

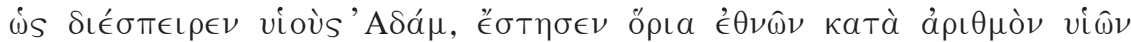

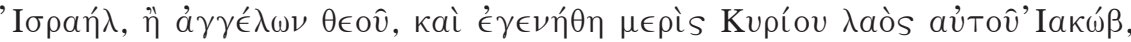

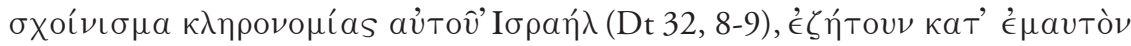

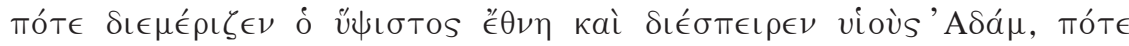

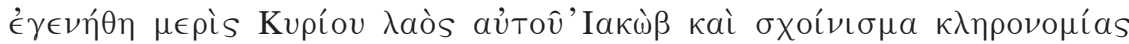

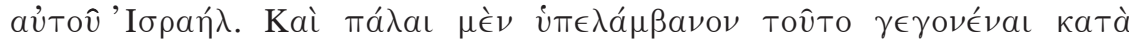

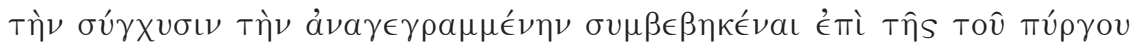

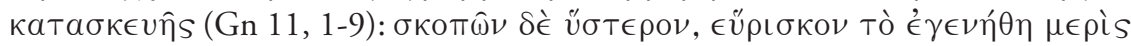

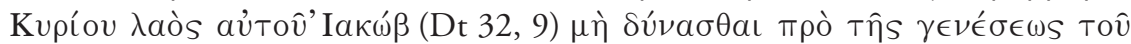

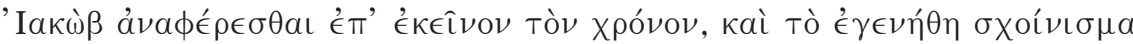

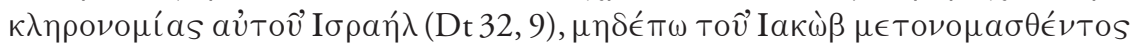

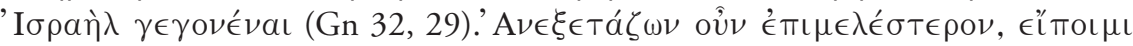

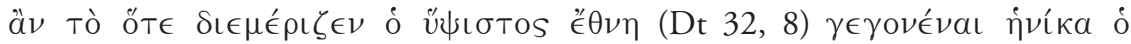

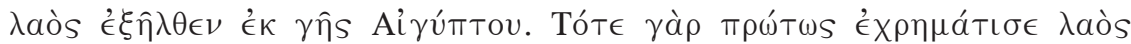

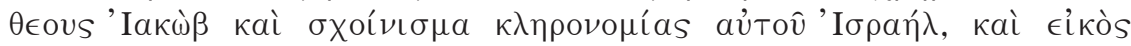

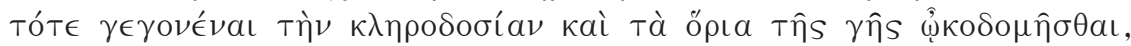

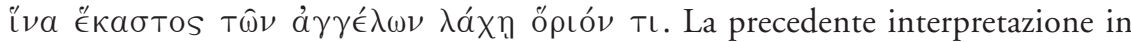
collegamento con Gn 11 è ancora attestata da CCV, 29 (30, 6-15); V, 31 (32, 20 26). Questo ed altri indizi fanno propendere per una datazione tarda delle nostre omelie, o almeno del ciclo di sermoni su Sal 77.

37 A parte H81Ps, i luoghi più significativi sono in H67Ps I, 5; H73Ps III, 5. Così, H67Ps I, 5 (f. 92r), a commento di 1 Cor 15, 42-43, afferma che colui che risorge non è più uomo, bensì 'dio', applicando il passo paolino prima al Salvatore uomoDio e poi all'uomo. A sua volta, secondo H73Ps III, 5 (f. 191r), Dio nella sua liberalità fa partecipare gli uomini alla stessa divinità, come vediamo dal fatto che si associ i nomi dei tre patriarchi Abramo, Isacco e Giacobbe in Es 3, 6.

38 Cf. H81Ps 1 (f. 361r), dove il lievito divino del Logos trasforma la 'pasta dell'uomo' facendola lievitare verso la divinità. Origene spiega qui il processo di divinizzazione 
l'Omelia I sul Salmo 80 attesta lo stesso tema, legandolo eloquentemente alla spiegazione del v. 3: "Intonate un salmo e suonate il timpano,

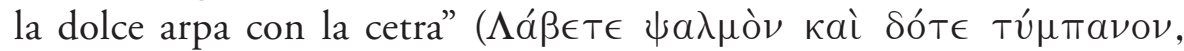

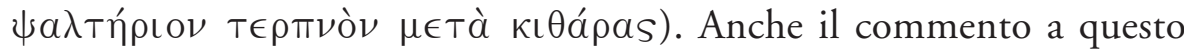
passo fonde significativamente gli aspetti dottrinali con quelli morali e spirituali. Dono di Cristo è rivelare il Padre e nel far ciò egli giunge a 'donare un salmo' riguardo a Dio: applicarsi alla conoscenza di Dio, dunque, significa 'ricevere un salmo' ${ }^{39}$. Non è difficile intravedere qui un'affinità con la visuale della preghiera come forma di 'teologia', secondo quanto Origene esplicita a commento del Padrenostro nel Trattato sulla preghiera ${ }^{40}$. Ma all'elemento conoscitivo e teoretico si unisce immediatamente quello pratico della risposta umana alla conoscenza di Dio. Essa consiste appunto nella santificazione di tutto quanto l'essere dell'uomo -corpo, anima e spirito-, col consueto richiamo a 1 Ts 5, 23, laddove i tre strumenti evocati da Sal 80, 3 corrispondono alle tre componenti dell'uomo (rispettivamente, timpano $=$ corpo, cetra $=$ anima

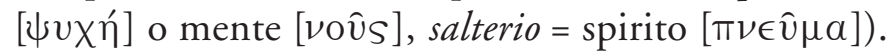

\section{LA VISUALE CRISTOLOGICA: SPUNTI DI CONFRONTO COL PERİ ARCHÔN}

Senza poter inseguire le molteplici piste d'indagine suggerite dalla collezione monacense, in linea col proposito iniziale vorrei soffermarmi

mediante le "tre misure di farina" della parabola evangelica (Mt 13, 33; Lc 13, 21) intese come le tre componenti dell'uomo: spirito, anima e corpo. Se non sorprende che lo spirito sia oggetto di divinizzazione, lo è invece per l'Alessandrino la divinizzazione dell'anima e soprattutto quella del corpo, perché esso ascenda glorificato al cielo.

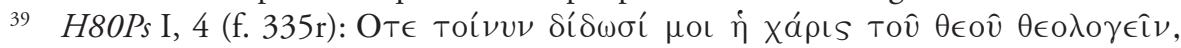

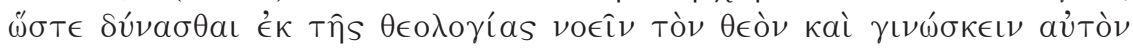

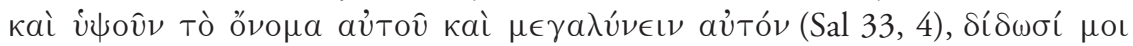

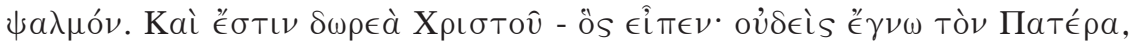

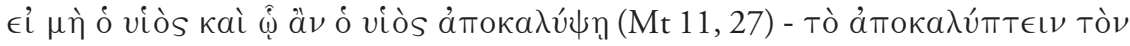

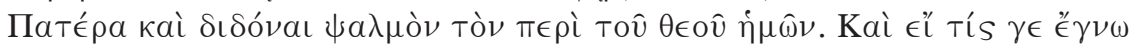

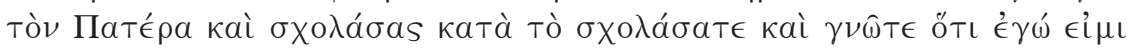

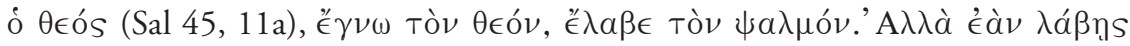

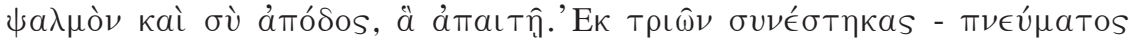

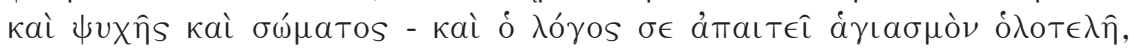

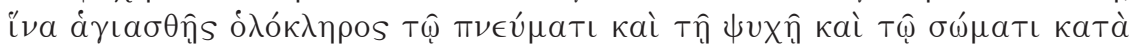

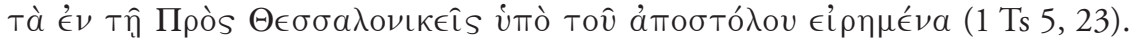

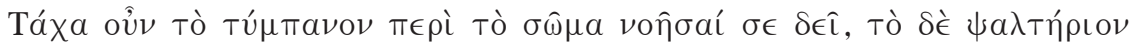

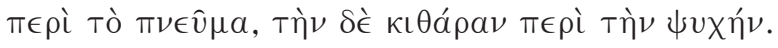

40 Cf. Orat XXIV. 
sull'elaborazione cristologica che essa presenta. Il suo luogo privilegiato è l'esegesi di Sal 15, testimonium di fondamentale importanza per la dottrina della resurrezione -come possiamo notare fin dall'uso che ne fa Pietro nel suo discorso il giorno di Pentecoste- e oggetto di due omelie che si ispirano entrambe alla traccia di At 2, 25-2841. Ma lo sviluppo organico di una dottrina della katabasis del Figlio a seguito della sua incarnazione, morte e discesa agli inferi, e della susseguente anabasis del Risorto fino alla dimora celeste si situa in un più vasto contesto di ri-

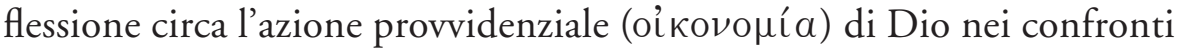
dell'uomo, attraverso la creazione e la redenzione. Per ricostruire tale sfondo teologico vale la pena considerare l'Omelia VI sul Salmo 77. In un certo senso, essa funge da preambolo dottrinale alla trattazione delle piaghe d'Egitto e dell'esodo di Israele, cui sono dedicate rispettivamente l'Omelia VII e l'Omelia VIII, mentre ripropone una visione del disegno salvifico affine al pensiero del Perì archôn. In particolare, data la prossimità dello sfondo scritturistico, si evidenziano analogie con la trattazione origeniana sul cuore indurito del Faraone nel Libro III ${ }^{42}$.

Questo ampio 'preambolo' dell' Omelia VI può ben essere definito un 'inno' all'amore misericordioso di Dio, che trae il suo avvio da Sal 77, 38a: "Ma egli è compassionevole, perdonerà i loro peccati e non distrug-

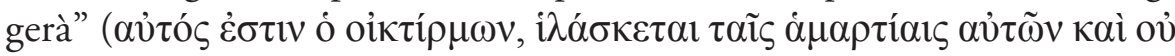
$\mu \eta ̀ ~ \delta i \alpha \varphi \theta \varepsilon i ́ p \eta)$. Per l'Alessandrino, che adduce al riguardo Sap 12, 10, Dio concede sempre un lasso di tempo all'uomo, perché si converta ${ }^{43}$. Come non ammirare in ogni generazione la pazienza e la misericordia di Dio verso gli uomini peccatori? Origene riformula l'idea, già esposta nell' Omelia sul Salmo 74, secondo cui Dio non fa mai sperimentare agli

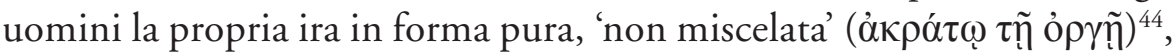
e ne offre la prova attraverso una rassegna del racconto biblico partendo dalla storia del diluvio col lunghissimo periodo concesso a Noè per la costruzione dell'arca che avrebbe dovuto smuovere i peccatori alla peni-

${ }_{41}$ Cf. H15Ps I, 2 (f. 3r); II, 1 (ff. 16v-17r).

42 Prin III, 1. Cf. anche Orat XXIX, 13-14.16.

43 Il richiamo a Sap 12, 10 è sfruttato in chiave analoga da H77Ps VII, 3 (f. 289v); HIer I, 1; VII, 1 .

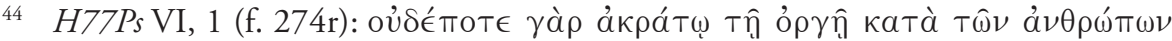

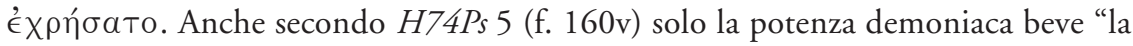
coppa pura" della malvagità, mentre per gli uomini è diversamente miscelata a seconda delle loro azioni buone e malvage. 
tenza. Un'altra prova sono gli angeli inviati a Lot prima della distruzione di Sodoma e Gomorra: tutti coloro che periscono, come la moglie di Lot, hanno dunque procurato loro stessi la propria morte. Anticipando poi una nota che ritroviamo nel commento dell'esodo contenuto nell' $O$ melia VIII, la misericordia divina si manifesta anche verso gli Egiziani: per l'Alessandrino, le piaghe inflitte loro da Dio hanno solo l'intento di convertirli, mentre i suoi castighi sono sempre le punizioni correttive di un Dio che è buono per natura ${ }^{45}$. Dunque, in ogni generazione tutte le operazioni divine sono ispirate dall'amore per l'uomo. Del resto, stando all' Omelia I sul Salmo 77, anche prima del dono della Legge a Mosè l'uomo non era abbandonato alla perdizione, ma era in grado, volendolo, di rispettare la legge naturale iscritta nella propria coscienza sull'esempio dei giusti Enoch e Abele: Dio infatti aveva disposto ciò che era conveniente per la salvezza di ognuno in ogni generazione, a condizione che l'uomo esercitasse rettamente il libero arbitrio ${ }^{46}$. Proseguendo il suo discorso nell' Omelia VI Origene aggiunge ancora una rilettura in chiave provvidenzialistica della carestia che colpisce l'Egitto, laddove la provvidenzialità è assicurata dalla venuta di Giuseppe nel paese. Come capita spesso nei suoi scritti quando si tratta di analizzare arditamente la Scrittura e affrontare questioni dottrinali ex mente Dei, egli ricorre ad une delle sue predilette prosopopee facendo parlare Dio in prima persona $^{47}$. È lo stesso artifizio retorico del quale l'Alessandrino si serve, ad esempio, nella spiegazione dell'indurimento di Faraone nel Perì archôn o nella quaestio sulla preghiera del Perì euchês ${ }^{48}$.

45 Per H77Ps VIII, 2 (f. 285v), la sorte fortunata toccata agli Israeliti non deve far dimenticare la bontà di Dio anche nei confronti degli Egiziani; Dio infatti castiga

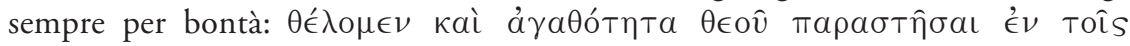

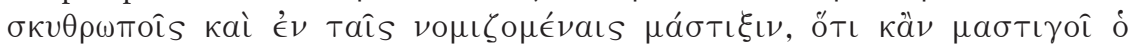

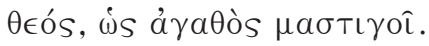

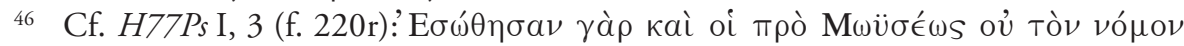

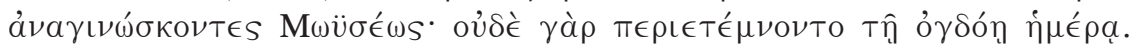

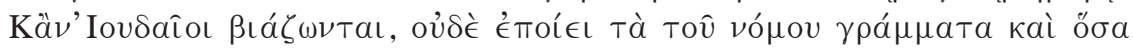

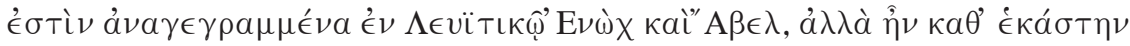

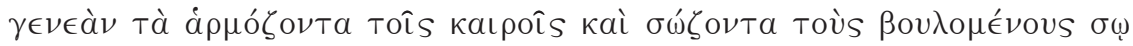
' $\zeta \in \sigma \theta a$.

47 Cf. A. VIllani, Origenes als Schriftsteller: ein Beitrag zu seiner Verwendung von Prosopopoiie, mit einigen Beobachtungen über die prosopologische Exegese, in Adamantius 14 (2008) 130-150.

48 Cf. Prin III, 1, 9.11; Orat VI, 4-5.

49 H77Ps VI, 1 (ff. 275v-276r). 


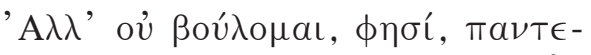

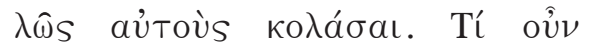

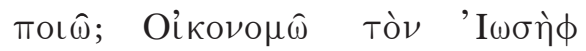

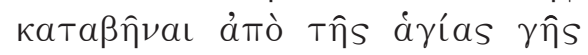

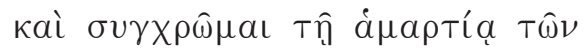

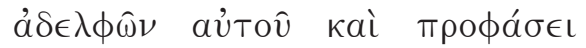

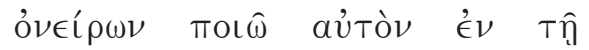

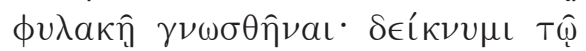

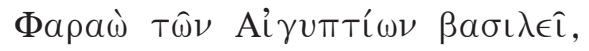

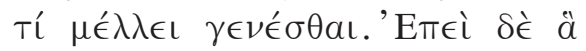

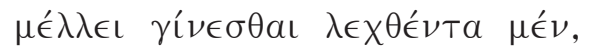

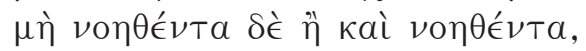

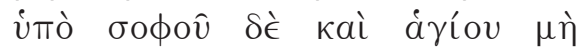

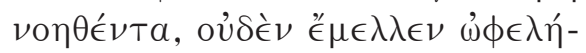

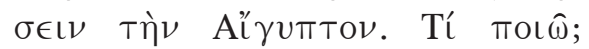

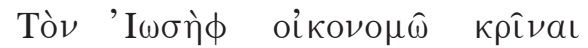

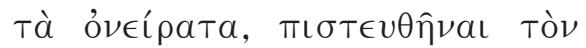

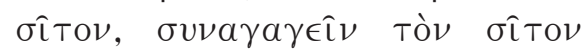

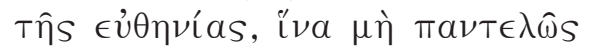
Sı $\alpha \phi \theta \alpha \rho \hat{n}^{49}$.
Ma io non voglio -dice- castigarli completamente. Cosa farò dunque? Disporrò che Giuseppe scenda dalla Terra Santa e mi servirò del peccato dei fratelli; col pretesto dei sogni farò sì che diventi noto nella prigione. Mostrerò al Faraone degli Egiziani che cosa starà per avvenire. Poiché ciò che deve avvenire, se verrà detto senza essere capito o se capito, non lo sarà da un saggio e santo, non porterà alcuna utilità agli Egiziani, che farò? Disporrò che Giuseppe interpreti i sogni, che raduni il grano dell'annona perché non sia distrutto completamente.

Quando l'umanità si era ormai privata per propria colpa della gloria di Dio $(\mathrm{Rm} 3,23)$, con la venuta del Figlio egli manda agli uomini un Salvatore e questi muore non solo per i giusti, ma anche per i peccatori, per "togliere i peccati del mondo" (Gv 1, 29). Né la misericordia divina nei confronti degli uomini cessa con il dono del proprio Figlio, poiché egli continua a manifestare la sua pazienza verso quanti l'offendono e insultano il suo nome, come fanno gli eretici. Se con questo cenno Origene richiama il motivo eresiologico antignostico e antimarcionita che contraddistingue le omelie, egli estende ancora la nozione dell'agire misericordioso di Dio alla stessa distruzione del mondo: essa si rende necessaria perché giunga il regno di Dio, ma anche in questo caso il suo agire va inteso come quello di un 'economo' provvidente, di un pedagogo saggio, di un 'medico' preoccupato di guarire il malato, cioè nei termini ben noti della concezione origeniana riguardo al Padre illustrata più direttamente dal Perì $\operatorname{archôn}^{50}$.

50 H77Ps VI, 1 (ff. 277r-v). Sul paradigma medico di Dio, si veda in particolare A.

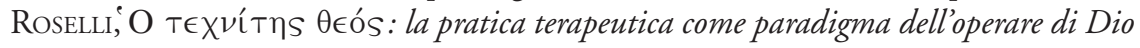
in Phil. 27 e PA III 1, in L. Perrone (a cura di), Il cuore indurito del Faraone. Origene 
Tracciato così l'orizzonte del disegno salvifico che ha al suo centro la venuta del Figlio nel mondo, la nota 'compassionevole' che lo sorregge in tutto il suo arco si palesa ab origine con una significativa riformulazione della 'compassione' misericordiosa di Dio dichiarata dalla celebre

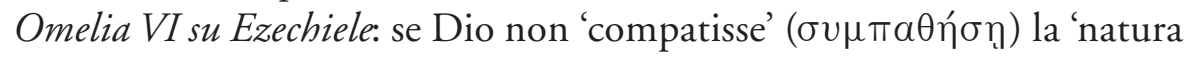

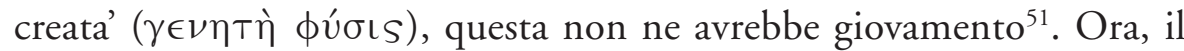
Figlio, in quanto immagine del Padre, imita sempre il suo modello; pertanto la sympatheia del Padre è condivisa dal Figlio che discende verso il cosmo, gli angeli e gli uomini, donandosi a ciascuno in relazione alle diversità di condizioni spirituali, e attuando a propria volta quel fonda-

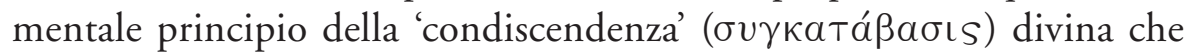
ispira sempre la condotta di Dio verso il mondo. Pertanto, le due Omelie sul Salmo 15, benché imperniate sul tema della discesa nella carne e dell'ascesa nella gloria, approfondiscono il legame del Figlio col Padre nel segno di una dipendenza che è ad un tempo subordinazione ma anche piena partecipazione all'essere di Dio. La dipendenza in chiave più prettamente subordinazionistica è messa in evidenza dall'interpretazione di Sal 15, 1b-2a: "Custodiscimi, Signore, perché in te ho sperato" ( $\Phi v$ -

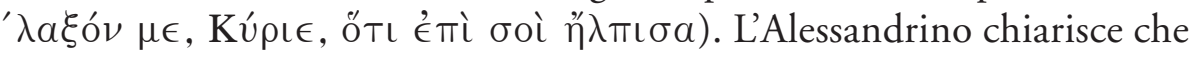
si tratta di una preghiera indirizzata al Padre dal Figlio, da identificarsi

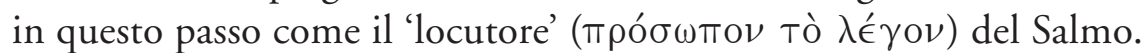

e il problema del libero arbitrio, Genova 1992, 65-83; S. Fernández, Cristo médico, según Orígenes. La actividad médica como metáfora de la acción divina (Roma 1999).

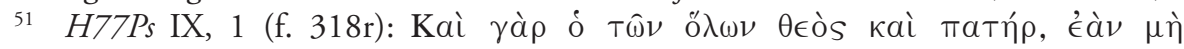

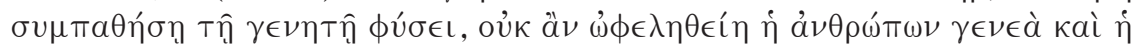

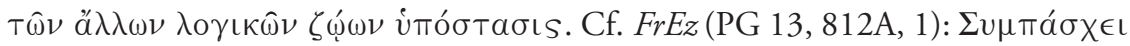

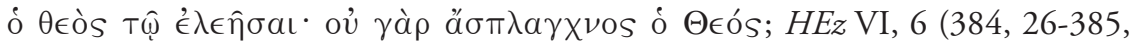
3): Pater quoque ipse et Deus uniuersitatis, longanimis et multum misericors (Sal 102, 8) et miserator, nonne quodammodo patitur? An ignoras quia, quando humana dispensat, passionem patitur humanam? Supportauit enim mores tuos Dominus Deus tuus, quomodo si quis supportet homo filium suum (Dt 1, 31). Igitur mores nostros supportat Deus, sicut portat passiones nostras filius Dei. Ipse Pater non est impassibilis. Si rogetur, miseretur et condolet, patitur aliquid caritatis et fit in iis, in quibus iuxta magnitudinem naturae suae non potest esse, et propter nos humanas sustinet passiones. Per un approfondimento, rimando a L. Perrone, "La passione della carità". Il mistero della misericordia divina secondo Origene, in Parola, spirito e vita, 29 (1994) 223-235; S. Fernández Eyzaguirre, 'Passio caritatis' According to Origen In Ezechielem Homiliae VI in the Light of Dt 1, 31, in Vigiliae Christianae, 60 (2006) 135-147; M.C. Pennacchio, Spiritus vivificat. Percorsi storico-letterari

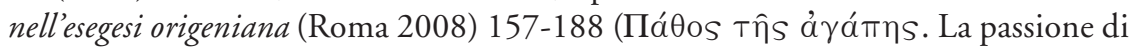
Dio in Clemente di Alessandria e Origene). 


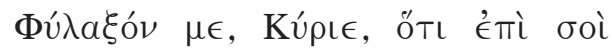

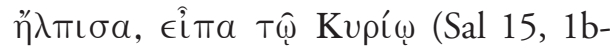

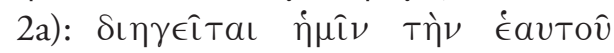

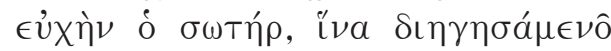

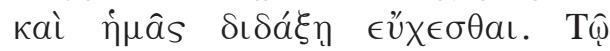

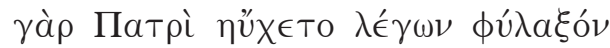

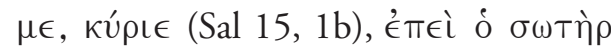

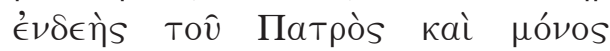

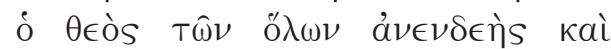

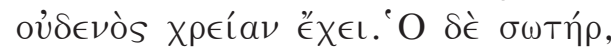

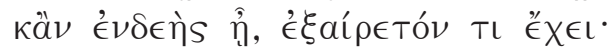

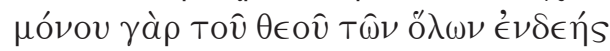

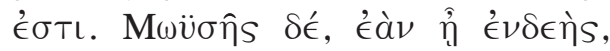

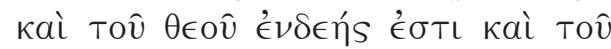

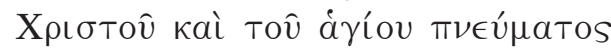

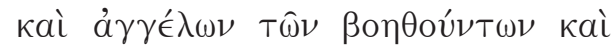

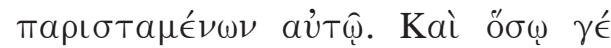

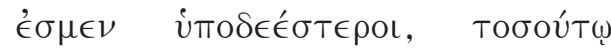

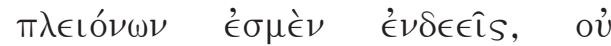

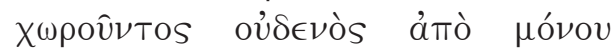

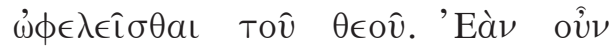

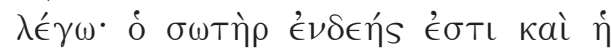

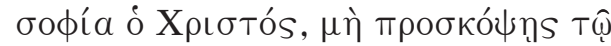

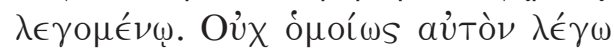

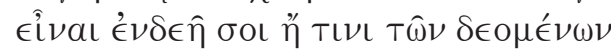

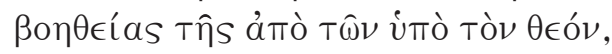

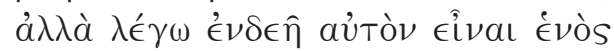
тôิ $\theta \in \mathrm{ov}$ кaì Патрós ${ }^{52}$.
"Custodiscimi, Signore, perche in te ho sperato, dissi al Signore" (Sal 15, 1b-2a): il Salvatore ci illustra la propria preghiera, affinché illustrandocela ci insegni anche a pregare. Infatti, dicendo "Custodiscimi, Signore”, egli pregava il Padre, poiché il Salvatore ha bisogno del Padre e solo il Dio dell'universo è privo di bisogni e non ha bisogno di nessuno. Tuttavia il Salvatore, anche se è bisognoso, possiede qualcosa di distintivo: egli ha bisogno unicamente del Dio dell'universo. Invece Mosè, se è bisognoso, abbisogna di Dio, di Cristo, dello Spirito santo e dell'aiuto e dell' assistenza degli angeli. Quanto più noi siamo inferiori, tanto più abbisognamo di molti, poiché nessuno è suscettibile di essere beneficato unicamente da Dio. Se dunque dico: il Salvatore è bisognoso, anche il Cristo Sapienza, non scandalizzarti per le mie parole. Io non lo dico bisognoso analogamente a te o a uno bisognoso dell'aiuto di quanti sono sotto Dio, bensì lo dico bisognoso dell'unico Dio e Padre.

Consapevole dell'arditezza dell'interpretazione, a causa delle evidenti implicazioni subordinazionistiche della sua visione gerarchica, Origene ne aggiunge subito una seconda di tipo ecclesiologico, premettendo la convinzione che sarà di sicuro più gradita, dal momento che indica come dicitore del passo Cristo in quanto Chiesa, cioè come il corpo mistico dei suoi fedeli ${ }^{53}$. Non è certo un caso che il Tractatus in Psalmum $X V$ di Gerolamo, che spesso riprende da vicino le due omelie origeniane,

52 H15Ps I, 3 (ff. 3v-4r).

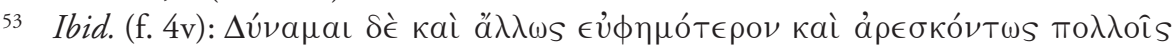

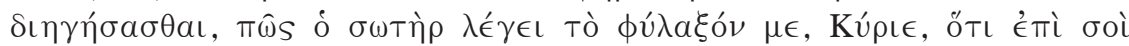


abbia ignorato la prima spiegazione presentando subito la seconda ${ }^{54}$. D'altra parte l'Alessandrino si premura di sottolineare l'unicità esclusiva del rapporto diretto che lega il Figlio al Padre. È vero che la definizione

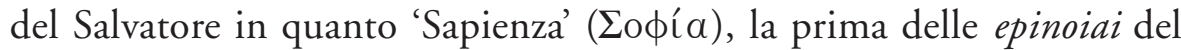
Figlio, fa capire come la situazione di dipendenza da Dio -nella quale si determina la dimensione del bisogno e la conseguente necessità della preghiera come richiesta di aiuto- investa la stessa persona del Verbo prima della sua incarnazione. Ma la 'recettività' per eccellenza del Figlio rispetto al Padre, fa sì che egli sia il solo a poterlo 'accogliere' o 'comprendere' direttamente (secondo quel verbo $\chi \omega \rho \in ́ \omega$ che connota per Origene la comunicazione di Dio e le modalità diverse della sua fruizione da parte delle creature $)^{55}$, mentre tutti gli esseri inferiori al Figlio hanno bisogno di tanti più intermediari quanto più cresce la loro condizione d'inferiorità. Mediante tale riflessione l'Alessandrino ribadisce dunque il modello eucologico del Trattato sulla preghiera col riferimento prioritario al Padre quale destinatario dell'orazione -com'è confermato dal seguito immediato dell'argomentazione ${ }^{56}$-, mentre il ruolo del Figlio quale mediatore ontologicamente subordinato tra Dio e gli esseri creati appare pienamente conforme alla prospettiva dottrinale tracciata dal Perì archôn.

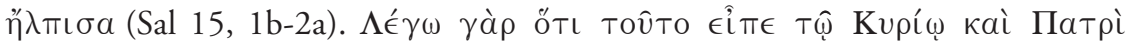

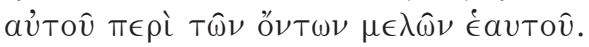

54 Hier., Tract. in Ps. XV, 1 (367, 101-103): Custodi me Domine, nisi enim custodieris meum corpus, ecclesiam quae in te sperat, in uanum uigilant qui custodiunt eam.

55 H67Ps II, 8 (f. $114 \mathrm{r}-\mathrm{v}$ ) spiega come Dio, che è 'incomprensibile', si renda

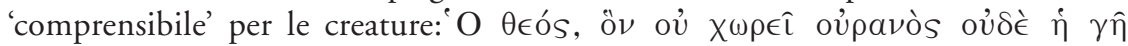

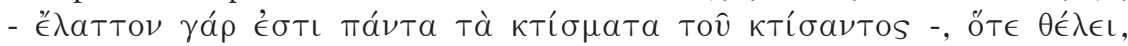

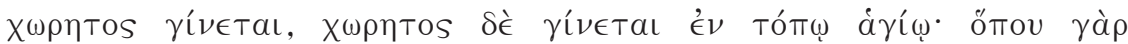

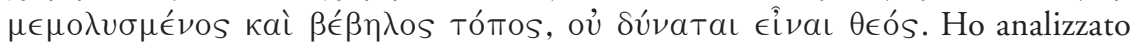

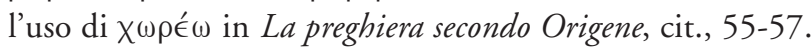

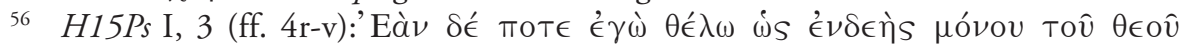

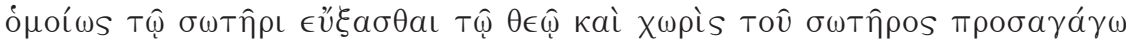

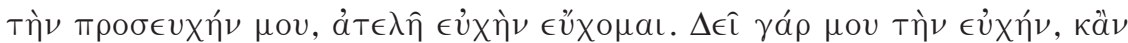

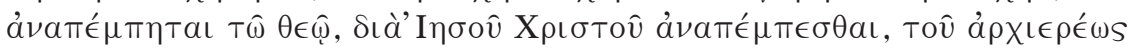

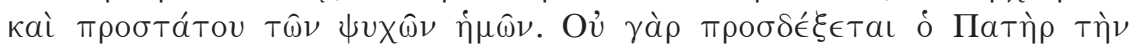

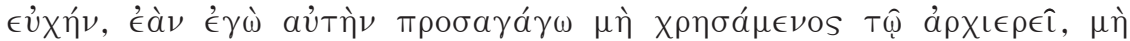

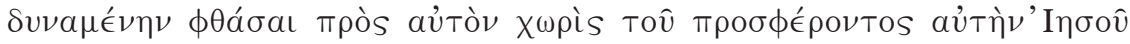
Xpıбтоิ. Cf. Orat XV, 2 (334, 18-21); XV, 4 (335, 19-22); e la mia analisi in La preghiera secondo Origene, cit., 133-140. 
Ne abbiamo, fra l'altro, una conferma dalla preminenza del titolo "Pri-

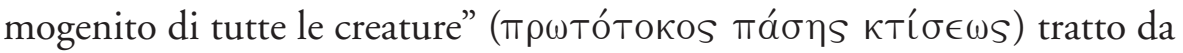
$\mathrm{Col} 1,15$, che le nostre omelie adoperano di preferenza per indicare la persona divina dell'Unigenito e distinguerla dal Figlio fatto uomo ${ }^{57}$. Come si è visto, esse operano l'identificazione tra il "Primogenito di tutte le creature" e la Sapienza, conformemente alla trattazione sul Cristo presente non solo nella dogmatica origenian $a^{58}$, ma più in generale negli scritti dell'Alessandrino. Riconoscendo in Col 1, 15 il 'Figlio di Dio' distinto dal 'Figlio dell'uomo', egli l'accosta anche allo Spirito santo in una posizione gerarchicamente superiore, come vediamo dall' Omelia III sul Salmo 73. Commentando Sal 73, 16b, in una forma diversa dal textus receptus della

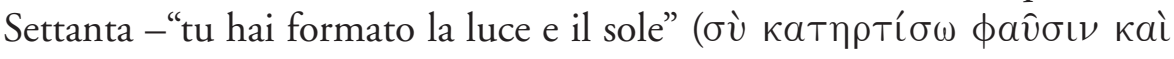

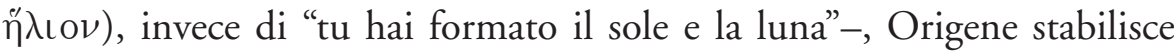
tropologicamente l'equivalenza 'luce' = 'Cristo', 'sole' = 'Spirito' in base ad una rilettura del racconto della creazione ${ }^{59}$. Ora, la creazione della luce (Gn 1,3) precede quella del sole (Gn 1,14) e come tale la 'luce' -in quanto 'Primogenito della creazione' - è più grande del 'sole' (dello Spirito) ${ }^{60}$.

Come si è già intravisto, la relazione del Figlio al Padre, pur nella 'subordinazione' ontologica, si dà nel segno dell'effusività di Dio e della partecipazione a Lui. Il Figlio che "è divenuto Dio a partire dalla divinità del Padre" -come ricorda l'Omelia I sul Salmo 15 chiarendo l'ornamento' apportato dal Logos agli angeli e agli uomini nella sua venuta nel mondo-, partecipa ininterrottamente della comunione con il Padre ${ }^{61}$. Egli è il suo 'cibo' e la sua 'bevanda', per cui anche le nostre omelie fanno proprio il motivo del Com-

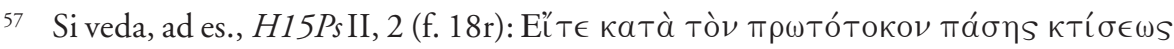

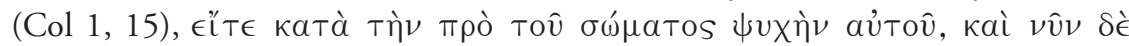

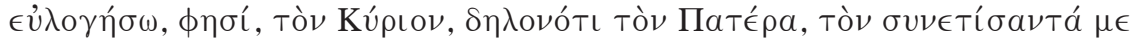
(Sal 15, 7a).

58 Cf. Prin I, 2, 1: Nec tamen alius est primogenitus per naturam quam sapientia, sed unus atque idem est.

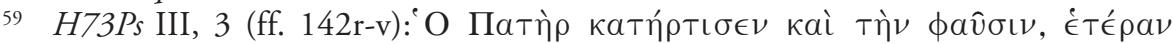

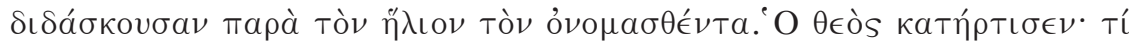

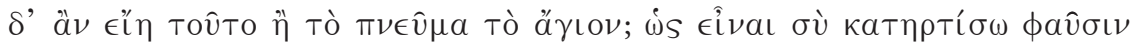

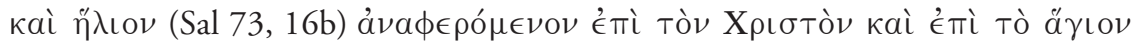

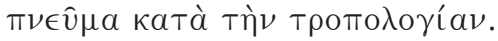

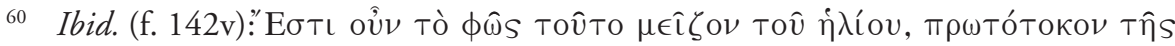

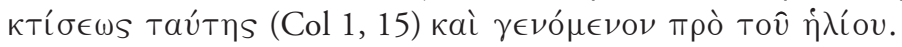

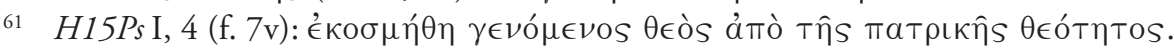


mento a Giovanni: il Figlio non trascura mai di 'nutrirsi' del Padre ${ }^{62}$. Il fondamentale discorso sui cibi spirituali, generalmente introdotto da Origene col riferimento privilegiato al "pane di vita" in Gv 6, non poteva non trovare materia anche nel commento dei nostri salmi, specialmente Sal 77 col racconto della manna. Ma il paradigma per eccellenza di ogni nutrimento spirituale è proprio quello rappresentato dalla relazione originaria e fontale tra il Padre e il Figlio. Infatti, vale anche per lui ciò che vale per tutte le creature razionali: il nutrimento è condizione e fonte della vita, e come noi 'mangiamo' e 'beviamo' il Logos, altrettanto fa il Figlio con il Padre ${ }^{63}$. Peraltro -come vediamo, in particolare, dalla spiegazione sul pane 'supersostanziale' ('́Tıó́oıOS) nel Perì euchês- la manducazione spirituale è intesa dall'Alessandrino anche come occasione di reciprocità della relazione spirituale ${ }^{64}$. Non a caso, dunque, ricordando la beatitudine del Figlio che 'beve' il Padre, egli accenna anche alla beatitudine del Padre che 'è bevuto' ${ }^{65}$. Tuttavia, l'accento batte piuttosto sulla 'letizia' ( $\epsilon \dot{u} \phi \rho o \sigma u ́ v \eta)$ molteplice del Figlio nel contemplare il volto del Padre ${ }^{66}$, mentre questi trova in lui il suo "luogo santo" per eccellenza, come osserva l' Omelia II sul Salmo 67.

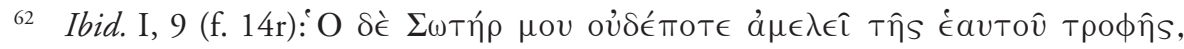

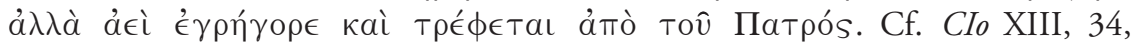

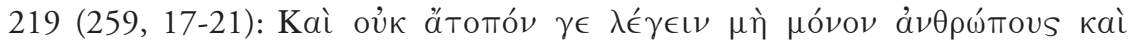

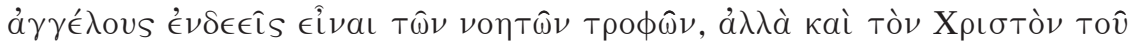

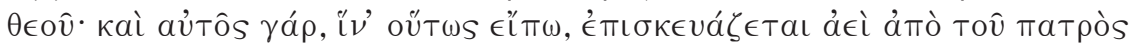

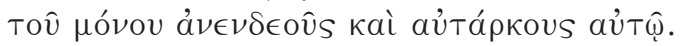

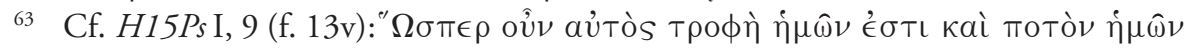

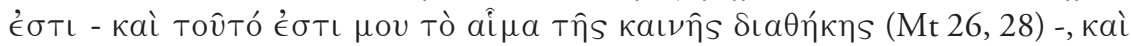

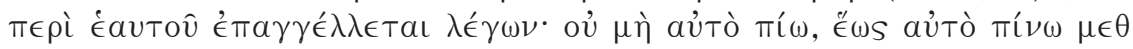

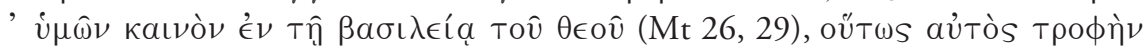
ÉX

64 Orat XXVII, 11 (370, 14-22). Si veda anche CIo XIII, 32, 198-199 (257, 1-8).

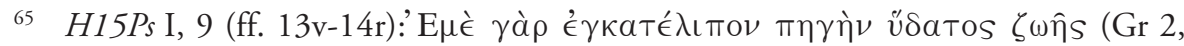

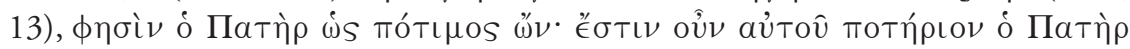

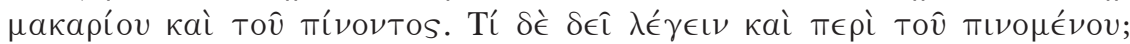

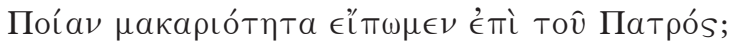

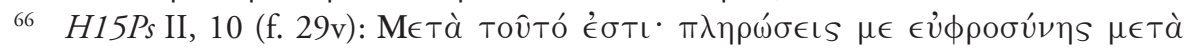

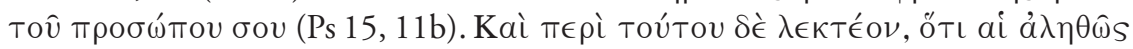

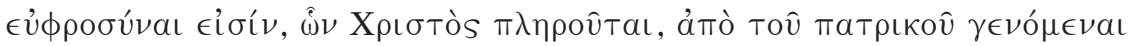

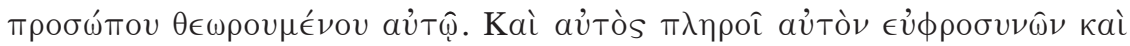

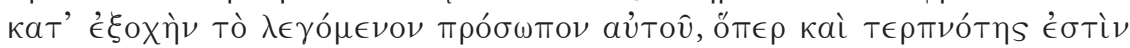

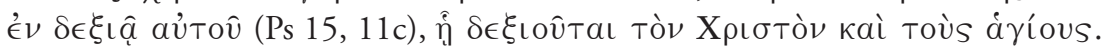




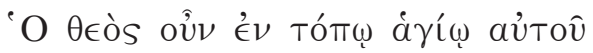
(Sal 67, 6b). Kaì $\epsilon \grave{l} \theta \in \operatorname{\lambda els,~}$

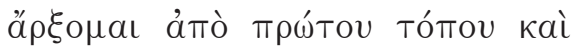

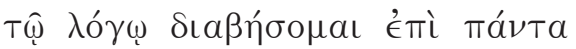

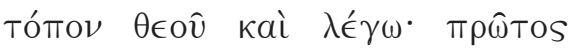

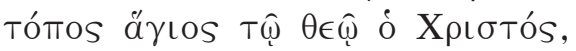

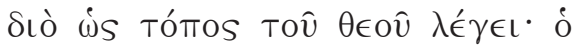

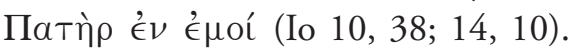

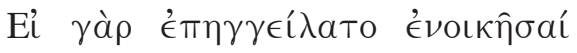

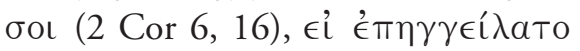

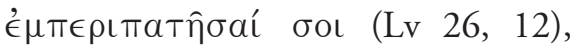

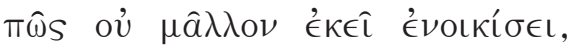

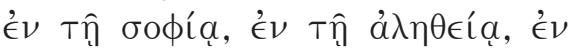

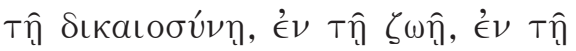

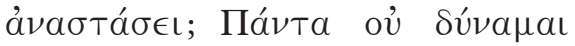

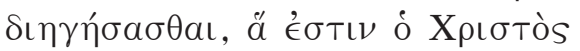

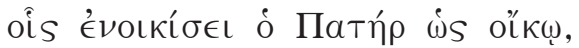

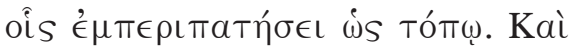

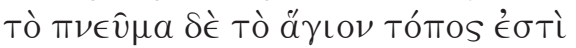

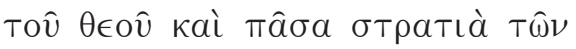

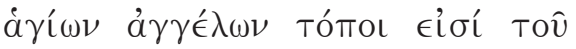

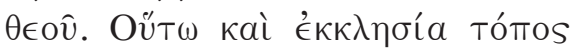

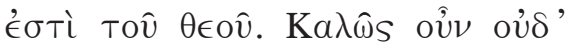

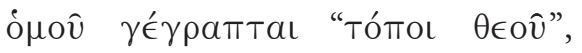

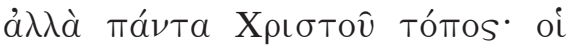

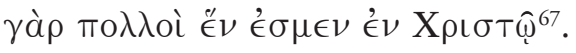

"Dio nel suo luogo santo" (Sal 67, 6b). Se vuoi comincerò dal primo luogo e passerò in rassegna col mio discorso ogni luogo di Dio dicendo: primo luogo santo per Dio è Cristo; perciò, in quanto luogo di Dio, egli dice: "Il Padre è in me" (Gv 10, 38; 14, 10).

Infatti, se (Dio) ha preannunciato di dimorare in te (2 Cor 6,16$)$, se ha preannunciato di passeggiare in te (Lv 26, 12), come non abiterà tanto più nella Sapienza, nella Verità, nella Giustizia, nella Vita, nella Resurrezione? Non posso illustrare tutto ciò che Cristo è, per coloro in cui il Padre dimorerà come in una casa, per coloro in cui passeggerà come in un luogo. $\mathrm{E}$ anche lo Spirito santo è un luogo di Dio, e tutta la schiera degli angeli santi sono luogo di Dio. Così anche la chiesa è luogo di Dio. Ora è stato scritto bene: non insieme luoghi di Dio' bensì tutto è luogo di Cristo; infatti noi molti siamo uno in Cristo".

In questo brano l'idea del Figlio come 'luogo di Dio' attira la dottrina delle epinoiai, tratto costitutivo della cristologia origeniana, approfondita sia nel Perì archôn sia specialmente nel Commento a Giovanni ${ }^{68}$, ma onnipresente negli scritti dell'Alessandrino ${ }^{69}$. Al tempo stesso nelle sue enun-

67 H67Ps II, 8 (ff. 114v-115r). La designazione di Cristo come 'luogo di Dio' trova

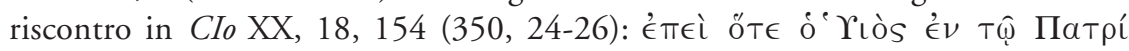

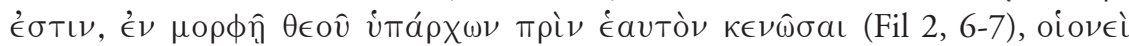

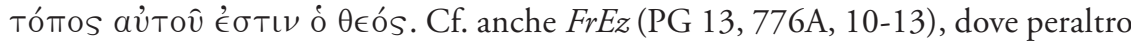

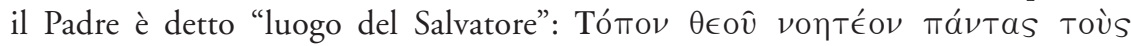

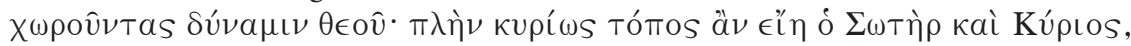

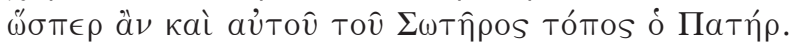

68 Prin I, 2, 1-4.13; II, 6, 1; CIo I, 21.

69 La dottrina è attestata anche da H36Ps I, 4 (f. 40r); H36Ps II, 4 (f. 47r); H73Ps I, 5 (f. 121v). Sulla cristologia origeniana si veda, in particolare, M. FÉDOU, La sagesse et le monde. Le Christ d'Origène (Paris 1994) spec. le pp. 233-269. 
ciazioni si avverte chiaramente una nota che forse non è sbagliato definire 'pancristica', strettamente collegata al ruolo mediatore del Cristo quale 'immagine di Dio' e a sua volta 'immagine’ egli stesso per le creature razionali, nel processo di divinizzazione che investe tutta quanta la creazione ${ }^{70}$.

Esso è tramato in radice dalla discesa del Logos che si fa carne e dall'ascesa del Risorto nella gloria che -come si è detto più volte- rappresentano il tema dominante delle due Omelie sul Salmo 15. Per Origene il dise-

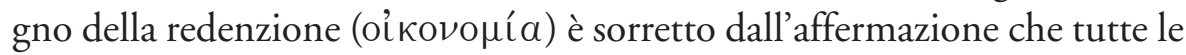
'regioni' ( $\chi \omega \rho i ́ a)$ del cosmo fino all'Ade hanno bisogno della venuta del Salvatore ${ }^{71}$; perciò egli fa dono di sé a ogni ordine di realtà, sia pure intendendo questa come ricompresa essenzialmente negli esseri razionali. In tal modo, la venuta del Figlio, in conformità con la kenôsis di Fil 2, 6-8, implica il suo abbassamento e assimilazione graduali a ogni loro condizione: egli "si fa tutto a tutti" (1 Cor 9, 22), angelo con gli angeli e uomo con gli uomini ${ }^{72}$. Non è chiaro in che termini l'Alessandrino intenda esattamente la metamorfosi angelica del Cristo, al di là del suo significato kenotico più immediato, ma in seguito la ribadisce con l'idea del suo trasfigurarsi alla portata di ciascuno ${ }^{73}$. In ogni caso, la visuale delle omelie trova conferma, ad esempio, nel Commento a Giovanni (se non anche nel testo originario

70 Cf. H15Ps I, 3 (ff. 5v-6r). P. Martens, Divinization, in The Westminster Handbook to Origen, ed. by J.McGucKIN (Louisville-London 2004) 91, afferma dunque giustamente che la 'divinizzazione' in Origene "describes God's gratious relationship to everything in the cosmos".

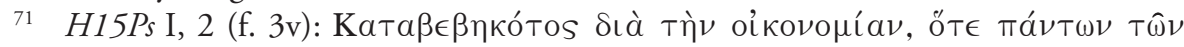

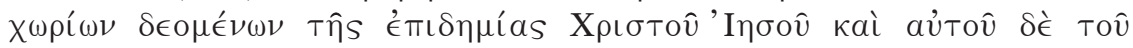

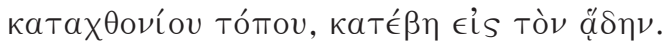

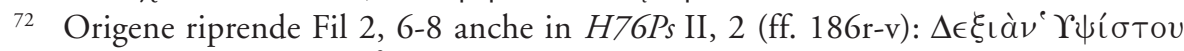

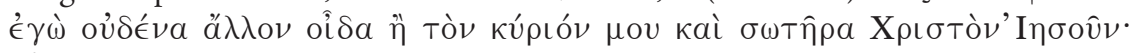

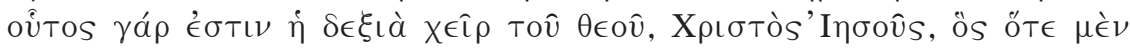

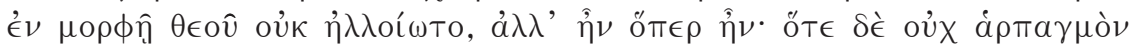

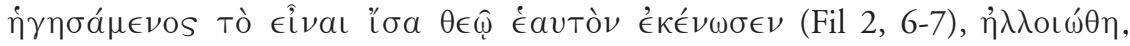

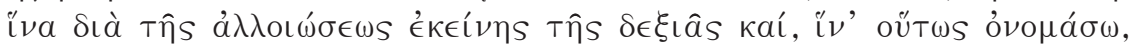

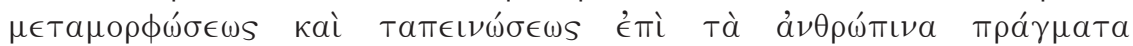

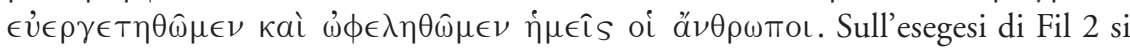
veda G. Bозтоск, Origen's Exegesis of the Kenosis Hymn (Philippians 2:5-11), in G. Dorival - A. Le Boulluec (a cura di), Origeniana Sexta: Origène et la Bible / Origen and the Bible (Leuven 1995) 531-547.

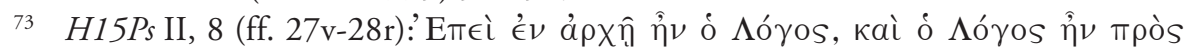

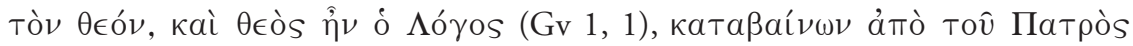

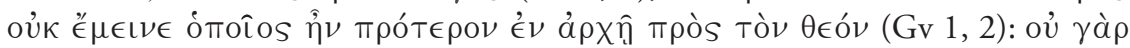

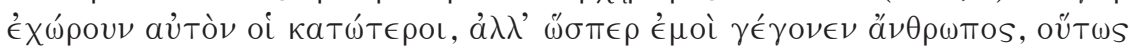


del Perì archôn $)^{74}$. Inoltre, l'azione salvifica attuata dal Figlio nel mondo angelico come nel mondo umano sembra finalizzata a conferire loro l'ornamento' della bellezza: con la sua discesa il Cristo 'adorna' non solo l'universo angelico ma anche quello degli uomini. Il 'pancristismo' si colora, per così dire, di 'pancosmismo', perché egli conferisce al 'cosmo' di angeli e uomini la sua pienezza e perfezione.

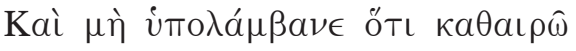

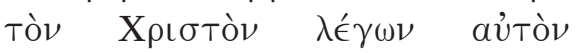

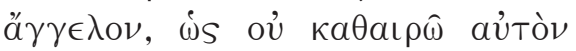

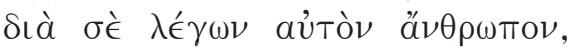

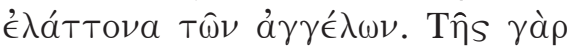

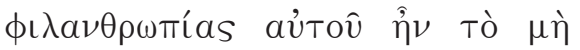

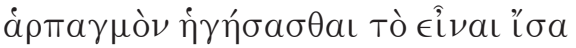

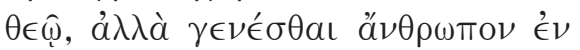

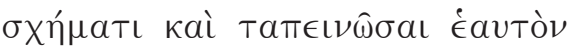

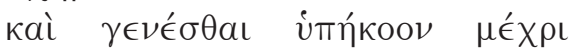

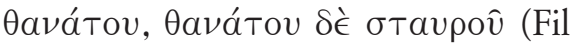

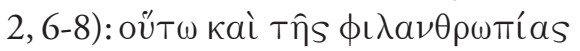

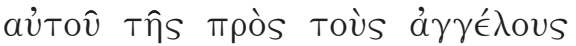

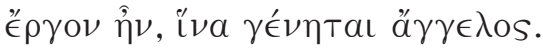

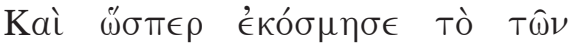

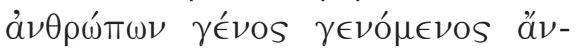

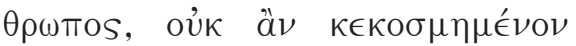

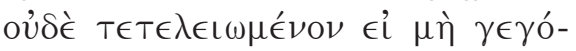
$\nu \in \mathrm{L}$ X

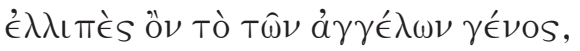

E non pensare che io cancelli Cristo dicendolo angelo, come non lo cancello dicendolo uomo per causa tua ed inferiore agli angeli. Era proprio della sua bontà non considerare un possesso geloso il suo essere uguale a Dio, bensì divenire in aspetto d'uomo, umiliare se stesso e rendersi obbediente fino alla morte, e alla morte di croce (Fil 2, 6-8). Così era opera della sua bontà verso gli angeli diventare angelo.

E come diventando uomo ha adornato la stirpe degli uomini, stirpe che non sarebbe stata adornata e perfezionata se Cristo non fosse divenuto uomo, così rimaneva ancora la stirpe degli angeli.

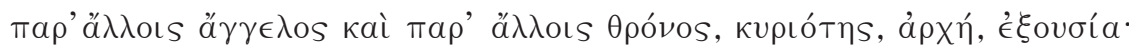

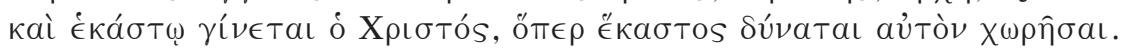

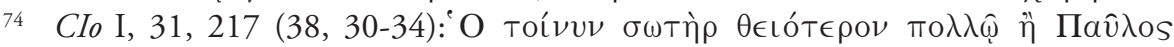

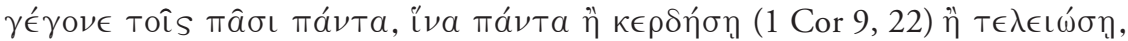

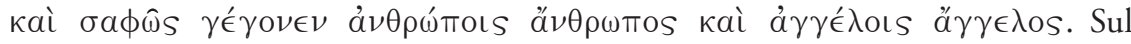
rapporto con Prin IV, 4, 5 si veda Origene. Commento a Giovanni, a cura di E. Corsini (Torino 1968) 178 n. 65. 


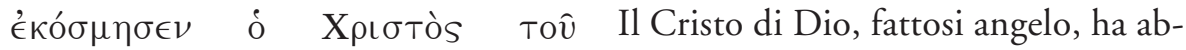

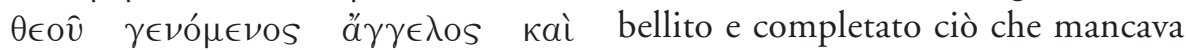

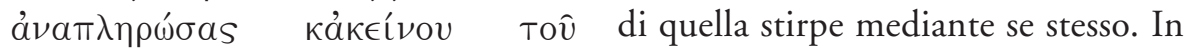

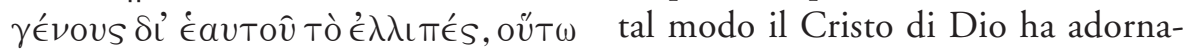

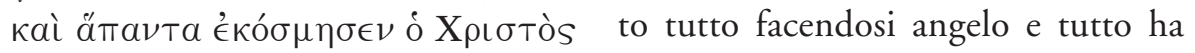

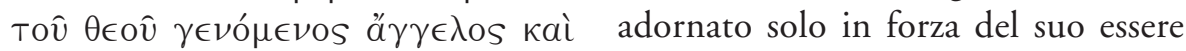

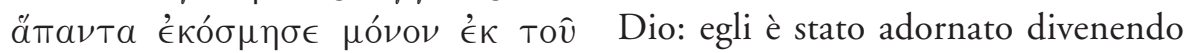

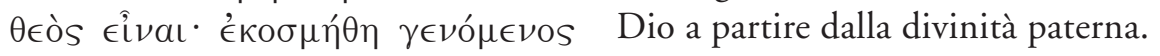

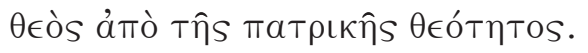

Se le nuove omelie tradiscono -come ho cercato di mostrare altro$\mathrm{ve}^{75}$ - un rinnovato interesse cosmologico, si comprende allora il significato di questo e altri passi, come l'intensa invocazione pronunciata da Origene nell' Omelia IV sul Salmo 77, perché il Logos quale 'pane celeste' discenda a tutte le creature e tutte alimenti, non solo in cielo e sulla terra ma anche sotto terra ${ }^{76}$. Infatti, se il Figlio rimane presso il Padre, coloro che si trovano 'in basso' rispetto a Lui non ne ricevono beneficio.

La metamorfosi umana del Figlio richiede allora che egli si faccia uomo in pienezza, cioè un 'essere composto' ( $\sigma u ́ \nu \theta \in T O S$ ) di pneuma, anima e corpo, secondo l'antropologia tricotomica di Origene fondata su 1 Ts 5, 23 e attestata a più riprese dalla collezione monacense, specie in relazione al discorso sulla deificazione ${ }^{77}$. Come nel Perì archôn e altrove, anche qui appare in primo piano il ruolo dell'anima di Cristo, non solo nella prospettiva dell'incarnazione, ma anche della discesa agli inferi di cui essa sola è protagonista. L'anima preesiste al corpo ed è 'istruita' dalla sua unione con il Logos prima della venuta nel mondo, come l'Alessandrino dichiara oscillando inizialmente nell'identificazione del locutore di Sal 15, 7a: "Benedirò il Signore che mi ha fatto comprende-

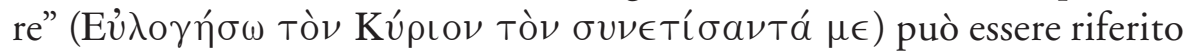
sia al Primogenito di tutte le creature (Col 1, 15) sia all"“anima prima

75 Rimando al mio contributo per il "Colloquium Origenianum Undecimum": The Find of the Munich Codex: A Collection of 29 Homilies on the Psalms (sub praelum).

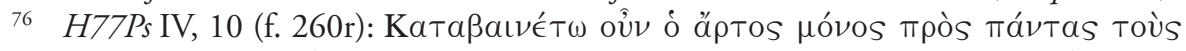

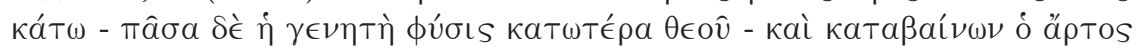

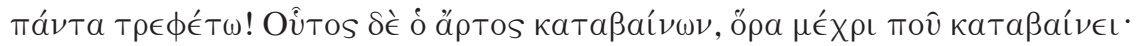

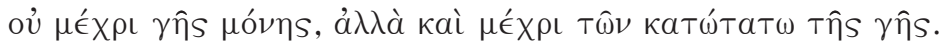

77 Cf. H77Ps VI (ff. 279v-280r); H80Ps I (ff. 335r-v); H80Ps II (f. 336v); H81Ps (f. 361v). 
del corpo"78. In realtà, la spiegazione che segue si focalizza sull'anima dell'uomo, soffermandosi con inedito sviluppo sui 'reni' di Sal 15, 7b:

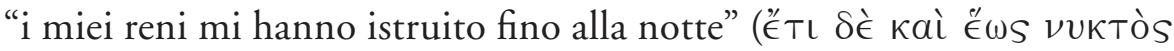

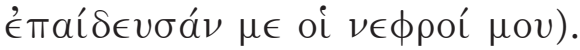

Origene chiarisce l'immagine dei 'reni' in maniera diversa dall'accezione più corrente nell'antropologia filosofica e adottata anche da lui in altra occasione, che li identifica simbolicamente con l'anima concupiscibile $^{79}$. Egli li sfrutta invece in chiave intellettiva, rivendicando un uso idiomatico delle Scritture che associa 'reni' e 'cuore ${ }^{\text {'80 }}$; pertanto i 'reni' rinviano al processo gnoseologico esplicantesi dalle "nozioni e germi dei

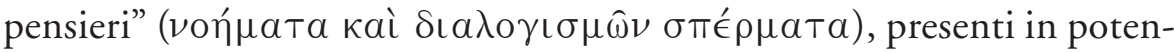
za nell'anima, fino all'atto del giudizio che matura sotto il controllo del 'cuore', l'organo egemonico della mente ${ }^{81}$. Ma è l'intima unione dell'anima con il Primogenito di ogni creatura che fa sì che l'anima di Cristo si unisca al corpo facendo da tramite con il Figlio. Di conseguenza i tratti cristologici dell' Omelia II sul Salmo 15 convergono con la dottrina sull'anima di Cristo nel Perì archôn. Lì l'anima, unita al Verbo di Dio e ripiena di Spirito, diventa Cristo, mentre il suo ardore per il Verbo la porta a superare la condizione dell'arbitrio fra bene e male, perché

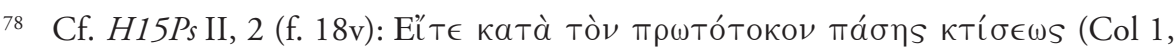

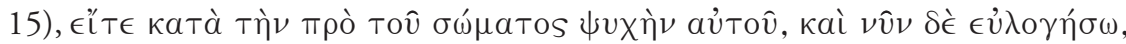

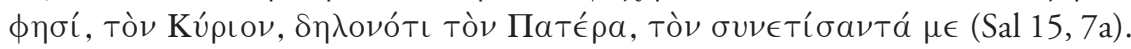

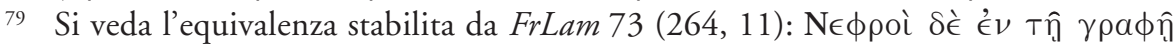

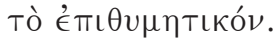

80 Sono numerose le trattazioni sui due termini associati, a partire dai loro riferimenti biblici (Ps 7, 10; Ier 17, 10; 20, 12). Cf. ad es. HIer XX, 9 (193, 32-34); Orat VIII, $2(317,13-16)$.

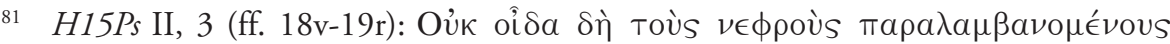

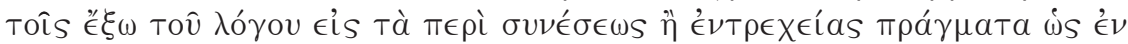

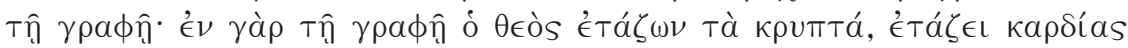

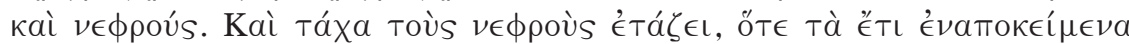

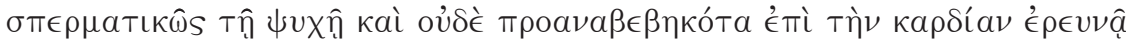
кaì $\mathfrak{\epsilon}_{\xi} \in \mathrm{T} \alpha \dot{\zeta} \in \mathrm{l}$. Gerolamo enfatizza il carattere distintivo dell'uso biblico; cf. Hier., Tr. XV, 7 (359-367): Nam quantum in memoria mea est, nec apud philosophorum quempiam nec apud rhetorum, nec apud poetas, nec apud ipsos quidem medicos, qui naturae corporum scientiam repromittunt, umquam legisse me noui, renes pro intellectibus et profunda cogitatione positos. Scriptura autem hac consuetudine utitur, ut quando uult secretum aliquid et arcanum uel mysticum demonstrare, dicat 'Scrutans corda et renes Deus' (Ps 7, 10); ut, cor secretum cum sit, secretiores cordis partes sint renes. 
la volontà buona diviene in lei come una seconda natura ${ }^{82}$. Anche per il nostro sermone l'impeccantia dell'anima di Cristo è legata al 'tesoro' deposto in essa dall'unione col Primogenito di tutte le creature. Essa è la sola quindi a non essere stata concepita nell'iniquità, secondo l'esegesi abituale di Sal 50, 7 sulle sordes dell'anima che l'Alessandrino ripropone anche qui ${ }^{83}$. È a questo punto che egli ricapitola la visuale dell'Incarnazione come intima unione tra l'anima e Dio in termini assai simili alla trattazione del Perì archôn (II, 6), grazie anche al ricorso a 1 Cor 6, 17 quale sua giustificazione scritturistica ${ }^{84}$.

82 Prin II, 6, 5 (144, 24-145, 4): Verum quoniam boni malique eligendi facultas omnibus praesto est, haec anima, quae Christi est, ita elegit diligere iustitiam, ut pro inmensitate dilectionis inconuertibiliter ei atque inseparabiliter inhaereret, ita ut propositi firmitas et affectus inmensitas et dilectionis inextinguibilis calor omnem sensum conuersionis atque inmutationis abscideret, ut quod in arbitrio erat positum, longi usus affectu iam uersum sit in naturam; ita et fuisse quidem in Christo humana et rationabilis anima credenda est, et nullum sensum uel possibilitatem eam putandum est habuisse peccati.

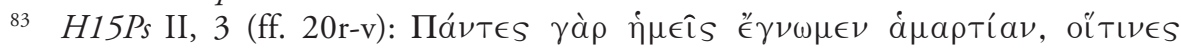

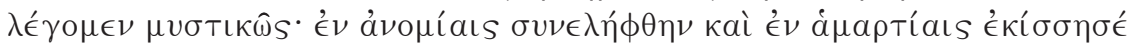

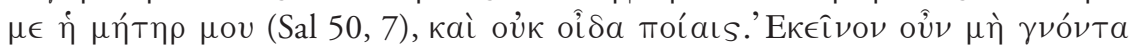

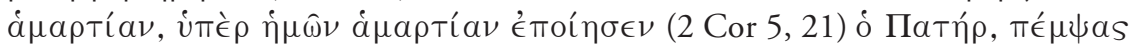

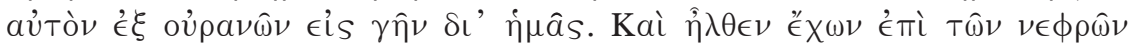

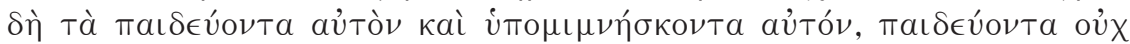

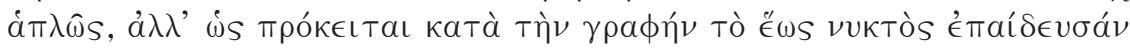

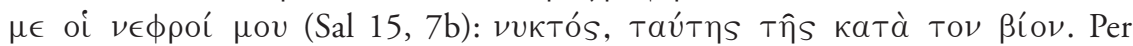
l'interpretazione di Sal 50, 7 si veda $C M t \mathrm{XV}, 23$ (417); CRm V, 9, 12 (440); CC VII, 50 (200, 19-25).

${ }^{84}$ Anche in CIoXXXII, 25, 326 (470, 20-24) Origene ricollega 1 Cor 6, 17 all'esegesi di Fil 2, 6 per sottolineare la piena unità del Figlio dell'uomo col Logos che si dà

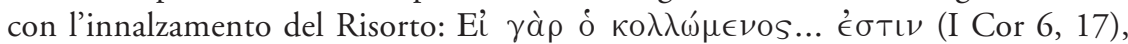

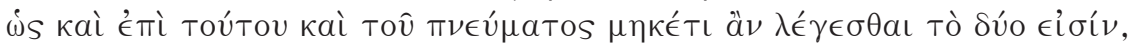

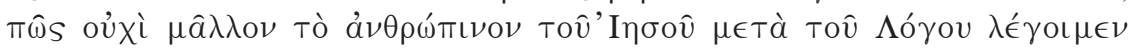

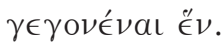


'H $\mathrm{H} \nu \theta \rho \omega \pi i ́ \nu \eta \quad \lambda \epsilon ́ \gamma \in \mathrm{l}$

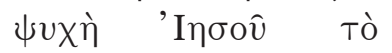

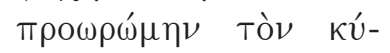

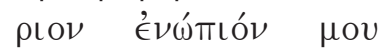
(Sal 15, 8a). Поồv Kúpıov; âpá $\gamma \in$ Tòv

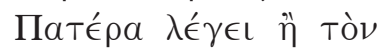

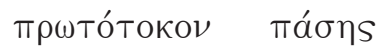

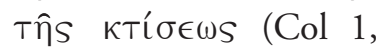

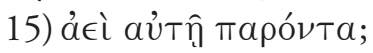

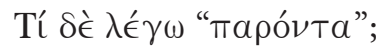

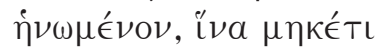

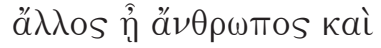

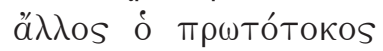

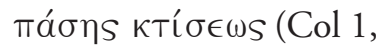
15). 'Еàv тробкółṇs

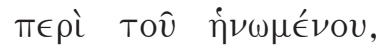

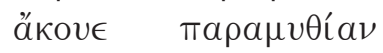

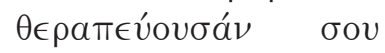

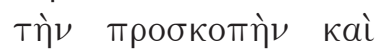

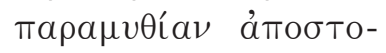

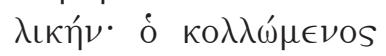

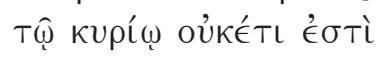

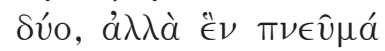
ÉoTLV (1 Cor 6, 17). Ế

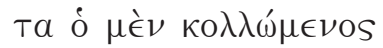

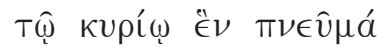

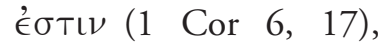

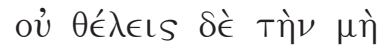

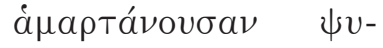

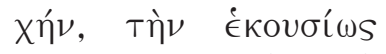

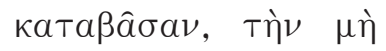

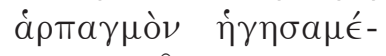
$\nu \eta \nu$ Tò Êेval ไ̌a $\theta \in \hat{\omega}$ (Fil 2, 6), ย๊ $v \quad \pi \nu \in \hat{v} \mu a$

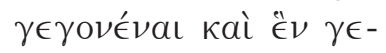

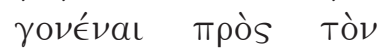

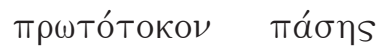
$\kappa T i ́ \sigma \in \omega S^{85}$;
È l'anima umana di Gesù che dice le parole: "Contemplavo il Signore davanti a me" (Sal 15, 8a). Quale 'Signore'? Chi designa dunque?: il Padre o il Primogenito di ogni creatura, che era sempre presente a lei? Ma perché dico 'presente'? Egli era unito a lei affinché non fosse più un altro l'uomo e un altro il Primogenito di ogni creatura (Col 1, 15). Se ti scandalizzi riguardo a colui che è unito, ascolta una prova che conforterà il tuo imbarazzo ed è una prova apostolica: "Colui che si unisce al Signore" non è più due "ma è un solo spirito" (1 Cor 6, 17). Pertanto "colui che si unisce al Signore è un solo spirito" (1 Cor 6, 17) e tu non vuoi che l'anima che non pecca, che è discesa volontariamente, che non ha ritenuto una preda essere uguale a Dio (Fil 2, 6), divenga un solo spirito e divenga una sola cosa col Primogenito di tutte le creature?
Illa anima, de qua dixit Iesus quia nemo aufert a me animam meam $(\mathrm{Gv}$ 10, 18), ab initio creaturae et deinceps inseparabiliter ei atque indissociabiliter inhaerens, utpote sapientiae et uerbo dei et ueritati ac luci uerae, et tota totum recipiens atque in eius lucem splendoremque ipsa cedens, facta est cum ipso principaliter unus spiritus, sicut et apostolus his, qui eam imitari deberent, promittit, quia qui se jungit domino, unus spiritus est $(1$ Cor 6,17$)[\ldots]$ Sed et unus spiritus esse cum Deo (1 Cor 6, 17) cui magis conuenit quam huic animae, quae se ita deo per dilectionem iunxit, ut cum eo unus spiritus merito dicatur ${ }^{86}$ ?

\footnotetext{
85 H15Ps II, 3 (ff. 20v-21r).

${ }^{86} \operatorname{Prin}$ II, 6, 3 (142, 4-10; 143, 14-17).
} 
L'insistenza sull'unione di Dio e uomo in Cristo non significa che Origene sia meno attento alla distinzione delle nature, tanto più che l'esegesi dei Salmi richiedeva frequentemente di distinguere gli aspetti umani e divini. In questo senso riflette sul corpo dell'Incarnato, sottolineando che non si tratta di un "corpo pneumatico", come vorrebbero avversari non dichiarati ma verosimilmente gnostici o docetisti, per i quali il corpo di Cristo sarebbe stato simile alla "sostanza del Logos" 87. Per contrasto, l'Alessandrino nel commentare il testimonium apostolico

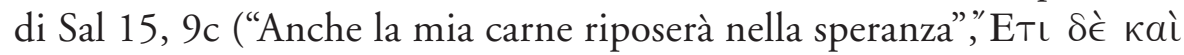

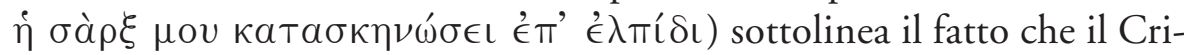
sto risorto, risalendo dagli inferi al cielo, reca con sé un "corpo terreno"

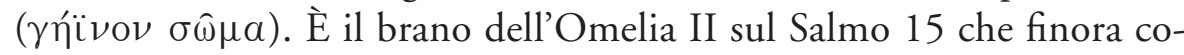
noscevamo nella traduzione latina di Rufino, ma che adesso ritroviamo nella più ampia cornice cristologica dell'omelia. La visione della carne gloriosa di Cristo, peraltro ancora segnata dalle tracce della passione,

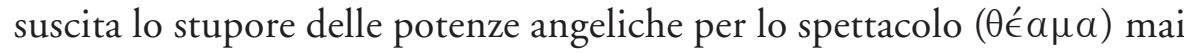
visto. Né Enoch né Elia possono essere addotti in senso stretto, per Origene, come esempio di santi ascesi al cielo nel loro corpo. Solo al Cristo può essere attribuito il titolo di "Primogenito dai morti" (Col 1, 18; Ap 1, 5), mentre il dialogo del Risorto con le potenze angeliche richiama il trionfo sulla morte nel corpo sofferente e crocifisso in maniera analoga al Commento a Giovanni.

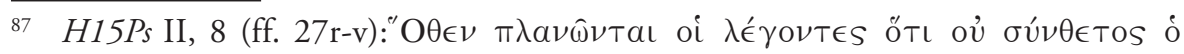

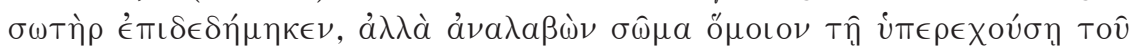

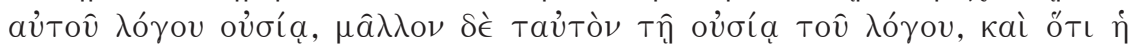

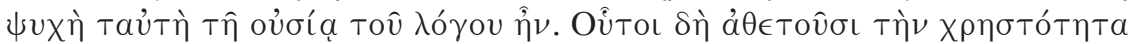

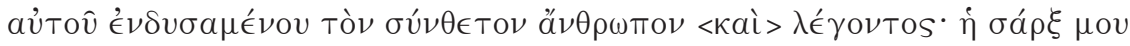

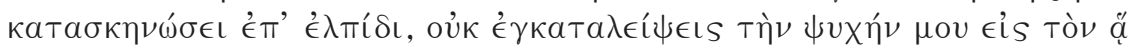

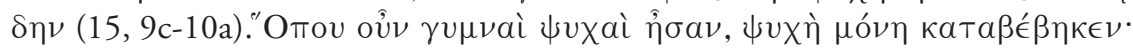

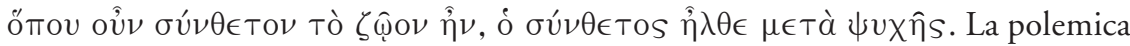

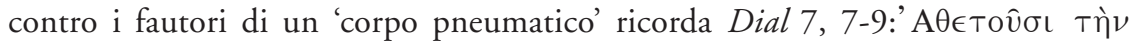

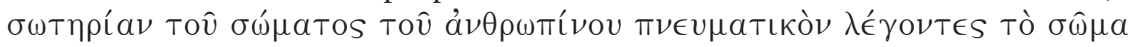
Tôิ $\sigma \omega t \hat{p} \rho \mathrm{s}$. 


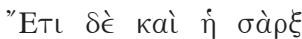

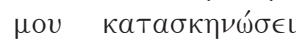

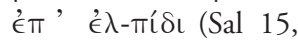
9c). 'O Kúptós pov 'I

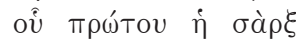

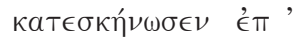

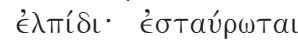

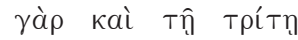

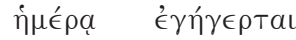

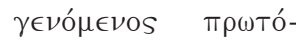

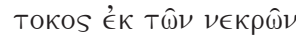
(Col 1, 18; Ap 1, 15) кaì

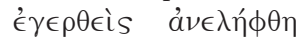
Eis oủpavòv kaì

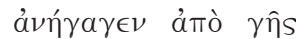

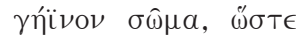
$\xi \in v^{\prime} \zeta \in \sigma \theta a \imath$ Toùs où-

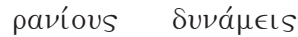

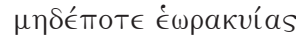

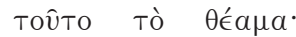
ба́рка àvaßaívovбav єis Tòv oúpavóv.

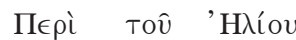

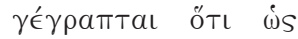

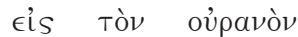
$\dot{a} v \in \lambda \eta ́ \phi \theta \eta(4 \operatorname{Rg} 2,11)$ кaì $\pi \epsilon \rho \grave{~ T o v ̂ ~ ' E v i ̀ x ~}$

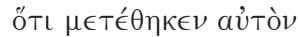
ò $\theta \in$ Ćs (Gn 5, 24; Eb $11,5)$, oưk El̄p Eis oúpavòv.

Прò тоरิ кupíov

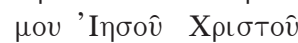
oủseis Eis oúpavòv àvaß́́ $\beta \eta \kappa \in \nu ~(G v 3,13)$.

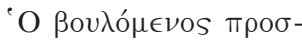

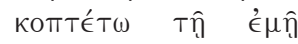

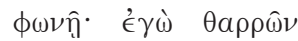

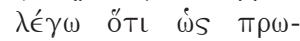
то́токо́s ÉбTLV ÉK $\tau \hat{\omega} \nu \nu \in \kappa \rho \hat{\omega} \nu(\mathrm{Col} \mathrm{1}, 18$;

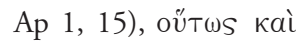
трйтоs ба́рка ảv-

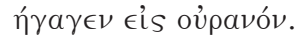

"Anche la mia carne riposerà nella speranza” (Sal 15, 9c). Il mio Signore Gesù dice ciò, il primo la cui carne ha riposato nella speranza. Infatti, fu crocifisso e al terzo giorno è risuscitato divenendo "primogenito dai morti” (Col 1, 18; Ap 1, 15). Dopo essere risu-scitato è stato assunto al cielo e ha recato dalla terra un corpo terreno, così da far stupire le potenze celesti che non avevano mai visto un tale spettacolo: una carne che ascende al cielo!

Riguardo a Elia sta scritto che "fu sollevato come verso il cielo" (4 Rg 2, 11) e riguardo a Enoch che "Dio lo trasferi" (Gn 5, 24; Eb 11, 5), ma non è detto "verso il cielo".

Prima del mio Signore Gesù Cristo "nessuno è asceso al cielo" (Gv 3, 13).

Si scandalizzi pure chi vuole per le mie parole, ma io dico con fiducia che come egli è il "primogenito dai morti" "(Col 1, 18; Ap 1, $15)$, così è stato anche il primo a portare la sua carne in cielo.
Pamphilus. Sed et in quinto decimo psalmo exponens illum uersiculum: Insuper autem et caro mea requiescet in spe (Sal 15, 9c), de carne Domini haec ait: Origenes. Dominus Jesus Christus haec dicit, cuius primo caro requieuit in spe. Crucifixus enim est et primogenitus ex mor-tuis factus (Col 1, 18; Ap 1, 15), et post resurrectionem adsumptus in caelum, secum terrenum corpus euexit, ita ut terrerentur et stupescerent caelestes uirtutes uidentes carnem ascendentem incaelum.

De Elia enim scriptum est, quia quasi in coelum adsumptus est (4 Rg 2, 11) et de Enoch quia translatus est (Gn 5, 24; Eb 11, 5), non tamen dictum est quia ascendit in caelum.

Offendatur qui uult ex nostro sermone, ego tamen cum omni fiducia adseuero, quia sicut primogenitus ex mortuis $(\mathrm{Col} 1$, 18; Ap 1, 15) est Christus, ita primus carnem euexit ad caelum.
Ma mentre egli si avanza vittorioso e trionfatore, con il suo corpo risuscitato da morte (ché non si possono intendere altrimenti le espressioni: "Non sono ancora salito al Padre" e "Io salgo al Padre mio"), allora alcune potenze celesti dicono: "Chi è costui che arriva da Edom, indossando un vestito rosso di Bosra, così pieno di forza?" (Is 63, 1), ma gli angeli che lo scortano dicono a quelli che sono posti a guardia delle porte celesti: "Principi, sollevate le vostre porte; porte eterne, inalzatevi, ed enterà il re della gloria" (Sal 23, 7). Ma [gli altri] ancora fanno domande (se è lecito esprimerci in questo modo), perché scorgono la sua destra insanguinata e lo vedono onusto dei risultati della sua gesta eroica: "Perché rosse sono le tue vesti, rosse come vinacce uscite da un torchio pieno d'uva pigiata?". Allora egli risponde: "Li ho pigiati” (Is 63, 2-3). 


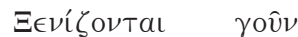

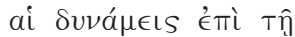
каเขทู iбторі́a, Öтเ

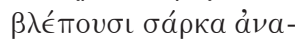

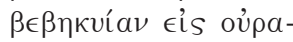

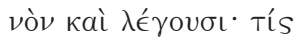

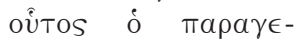

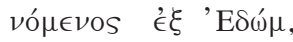

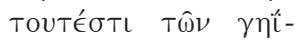
$\nu \omega \nu, \quad \epsilon \rho v ́ \theta \eta \mu \alpha$ i $\mu \alpha-$ TíwV (Is 63, 1a);

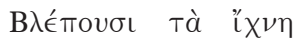
тоv aïnatos kaì

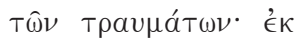
Boбóp, тท̂s баркós,

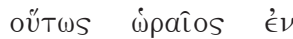

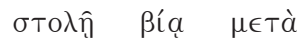

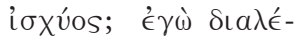
ropal (Is 63, 1b-c). Tís oûtos; Kaì oủk

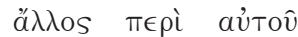
ámокрі́veтаᄂ, $\dot{a} \lambda \lambda$, aủTòs $\mu \in T a ̀$ íxúos.

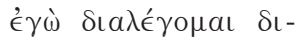

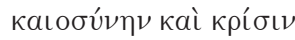

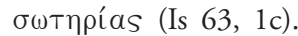

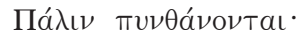

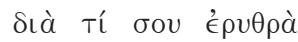
тà iнátıa кaì Tà

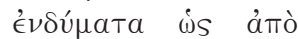

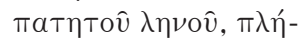

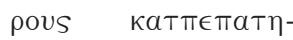
$\mu$ $\mu$ év (Is 63, 2-3); Eî

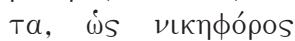

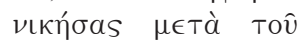

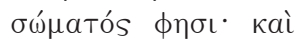

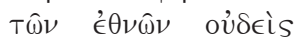
$\mu \in T^{\prime}$ '́ $\mu о \hat{~ \kappa a i ̀ ~ \kappa a t \epsilon-~}$

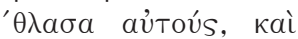

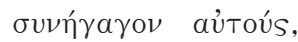

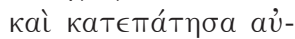
Toùs Eis $\gamma \hat{\eta} \nu$ (Is 63, $3)^{88}$.
Pertanto le potenze si stupiscono per la visione inedita, poiché vedono una carne che sale al cielo e dicono: "Chi è costui che viene da Edom -cioè dalle realtà terrene-, il rossore delle sue vesti?" (Is 63, 1a). Vedono le tracce del sangue e delle ferite: "da Bosor -dalla carne-, così bello nella sua veste, con vigore e forza? Io parlo" (Is 63, 1b-c).

"Chi è costui"? E non altri risponde riguardo a lui, ma è lui stesso a dire con forza: "Io parlo della giustizia e del giudizio di salvezza" (Is 63, 1c).

Di nuovo lo interrogano: "Perché rosse sono le tue vesti e i tuoi abiti, rosse come vinacce uscite da un torchio pieno d'uva pigiata? (Is 63, 2-3)".

Poi, come un vincitore che ha vinto col suo corpo, dice: "Nessuno delle nazioni è con me, io ho gareggiato contro di loro, li ho radunati e li ho pigiati sulla terra" (Is 63, 3).
Denique nouitate Egli aveva veramenipsa perterrentur te bisogno di lavacaelestes uirtutes, re "nel vino la sua quia quod nun- veste e nel sangue quam antea uider- dell'uva il suo manant nunc uidebant to" (Gn 49, 11). Incarnem ascenden- fatti, avendo preso le tem in caelum et nostre debolezze ed propterea dicunt: essendosi addossato Quis est iste qui le nostre malattie venit de Edom (Mt 8, 17; Is 53, 4), -id est a terrige- avendo preso su di nis- Rubor uesti- sé il peccato di tutto mentorum eius ex il mondo e avendo Bosor (Is 63, 1a)? beneficato tanti, egli Videbant enim ue- ricevette forse allora stigia uulnerum in un battesimo più corpore eius ex Bo- grande di quanto sor, id est in carne gli uomini possano suscepta $^{89}$. concepire $^{90}$.

${ }^{88}$ H15Ps II, 8 (ff. 26r-v).

89 Fr. lat. ex Pamph., Apol. 142-145 (228-232 Amacker-Junod).

90 CIo VI, 56, 288-289 (164, 29-165, 9 [trad. tratta da Origene. Commento a Giovanni, a cura di E. Corsini, cit., 372-373). Origene ha commentato anche altrove Is 63; 
L'ampio commento di Is 63 si conclude nella nostra omelia col v. 7: a seguito del dialogo col Risorto, le potenze angeliche "si ricordano della misericordia del Signore", della sua bontà in tutto ciò che egli ha fatto per l'uomo. Ma dal confronto sinottico col passo corrispondente del Commento a Giovanni si direbbe che l'Omelia II sul Salmo 15 attribuisca particolare risalto al protagonismo esclusivo del Risorto, senza menzionare l'intervento diretto delle potenze angeliche che lo scortano. Più importante è però l'enfasi sul ruolo del corpo, accompagnata da un deciso ridimensionamento di eventuali antecedenti veterotestamentari (Enoch ed Elia) che rende assolutamente unica l'ascesa trionfale della carne di Cristo, ancora segnata dalle sofferenze della passione. A sua volta Rufino ha ripreso fedelmente il passo, che gli serviva per scagionare l'Alessandrino dall'accusa di negare la resurrezione della carne. Tuttavia, ha tralasciato una nota significativa, con la quale possiamo chiudere questa prima riflessione sull'apporto cristologico delle nuove omelie. Per due volte Origene adopera l'espressione "il mio Signore" in riferimento a Gesù Cristo - un tratto che Rufino non ha ripreso ma che conosciamo bene anche dagli altri scritti ${ }^{91}$. Esso però

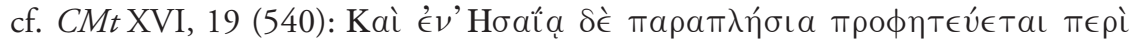

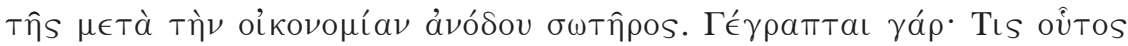

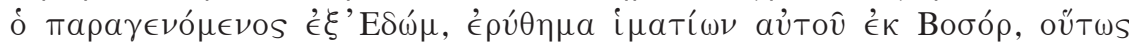

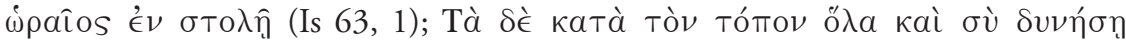

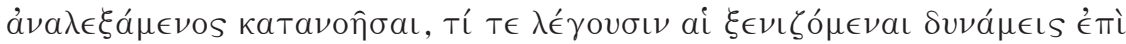

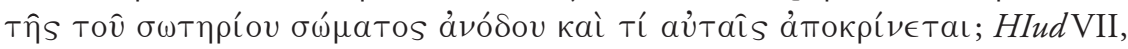
2 (508): Beatus est de quo eunte ad caelum dicunt angeli illam propheticam uocem: Quis iste est qui adscendit ex Bosor (Is 63, 1)? Hoc est qui de carne ad caelum adscendit, quis est qui adscendit ex Bosor rubor uestimentorum eius (Is 63, 1)?, in rubore uestimentorum cruorem sanguinis designantes. Beatae ergo sunt animae quae sic Christum sequuntur quomodo eas Christus praecessit.

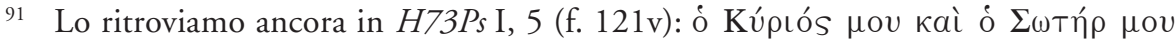

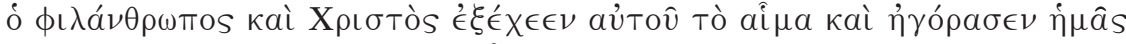

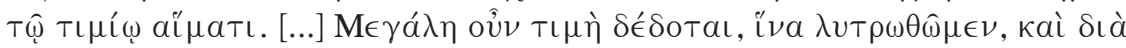

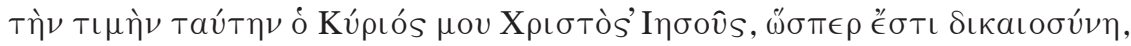

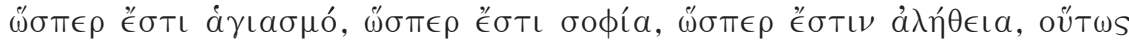

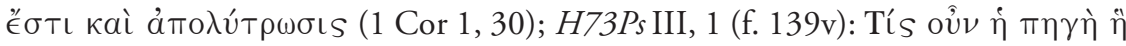

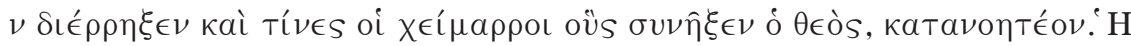

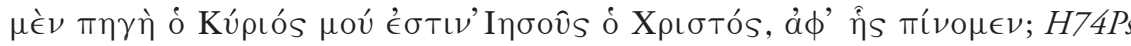

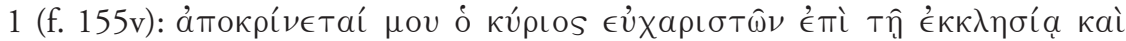

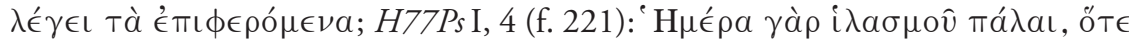

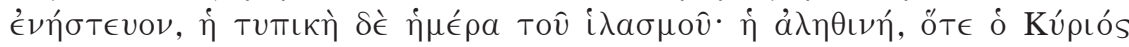

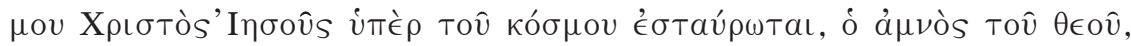


non è affatto secondario, perché pone la riflessione cristologica delle nuove omelie, ripercorsa qui in alcuni esempi più significativi, nella luce di un'intima devozione a Cristo.

ó al̆

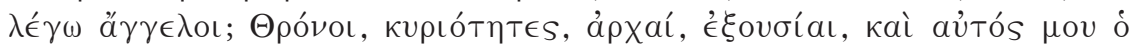

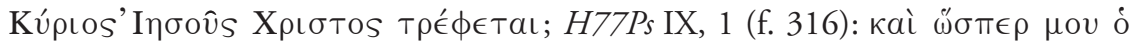

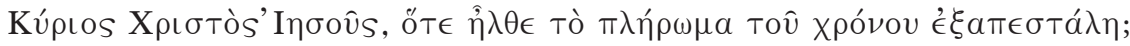

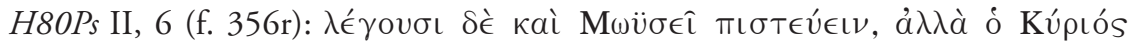

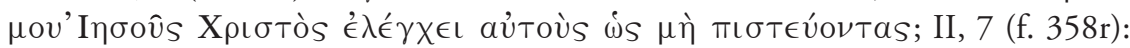

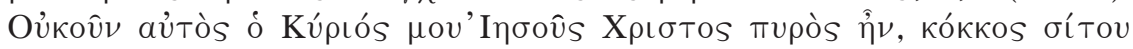

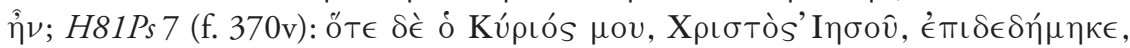

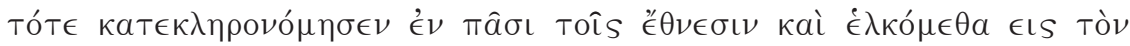

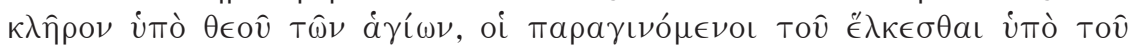

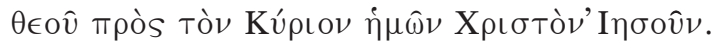

